\title{
Oxidation-specific epitopes are dominant targets of innate natural antibodies in mice and humans
}

\author{
Meng-Yun Chou, ${ }^{1}$ Linda Fogelstrand, ${ }^{1}$ Karsten Hartvigsen, ${ }^{1}$ Lotte F. Hansen, ${ }^{1}$ Douglas Woelkers, ${ }^{2}$ \\ Peter X. Shaw, ${ }^{1}$ Jeomil Choi, ${ }^{1,3}$ Thomas Perkmann, ${ }^{4,5}$ Fredrik Bäckhed, ${ }^{6}$ Yury I. Miller, ${ }^{1}$ \\ Sohvi Hörkkö, ${ }^{1}$ Maripat Corr, ${ }^{1}$ Joseph L. Witztum, ${ }^{1}$ and Christoph J. Binder ${ }^{1,4,5}$

\begin{abstract}
${ }^{1}$ Department of Medicine and ${ }^{2}$ Department of Reproductive Medicine, UCSD, La Jolla, California, USA. ${ }^{3}$ Department of Periodontology, Pusan National University, Pusan, Republic of Korea. ${ }^{4}$ Center for Molecular Medicine (CeMM) of the Austrian Academy of Sciences and ${ }^{5}$ Department of Medical and Chemical Laboratory Diagnostics, Medical University of Vienna, Vienna, Austria.

${ }^{6}$ Sahlgrenska Centre for Cardiovascular and Metabolic Research, Wallenberg Laboratory, University of Göteborg, Göteborg, Sweden.
\end{abstract}

\begin{abstract}
Atherosclerosis is a chronic inflammatory disease characterized by the accumulation of oxidized lipoproteins and apoptotic cells. Adaptive immune responses to various oxidation-specific epitopes play an important role in atherogenesis. However, accumulating evidence suggests that these epitopes are also recognized by innate receptors, such as scavenger receptors on macrophages, and plasma proteins, such as C-reactive protein (CRP). Here, we provide multiple lines of evidence that oxidation-specific epitopes constitute a dominant, previously unrecognized target of natural Abs (NAbs) in both mice and humans. Using reconstituted mice expressing solely IgM NAbs, we have shown that approximately $30 \%$ of all NAbs bound to model oxidation-specific epitopes, as well as to atherosclerotic lesions and apoptotic cells. Because oxidative processes are ubiquitous, we hypothesized that these epitopes exert selective pressure to expand NAbs, which in turn play an important role in mediating homeostatic functions consequent to inflammation and cell death, as demonstrated by their ability to facilitate apoptotic cell clearance. These findings provide novel insights into the functions of NAbs in mediating host homeostasis and into their roles in health and diseases, such as chronic inflammatory diseases and atherosclerosis.
\end{abstract}

\section{Introduction}

Although hypercholesterolemia is necessary for the initiation and progression of atherosclerosis, there is now abundant evidence that immune mechanisms are also central to all phases of lesion development (1-3). We and others have documented that, among several suggested immunogens present in the atherosclerotic plaque, oxidation-specific epitopes, as occur in oxidized LDL (OxLDL), are immunodominant. In turn, these lead to profound immune responses, including autoantibody generation, that modulate lesion formation (4).

Many of these responses are adaptive in nature, responding to the myriad of new moieties generated in response to the complex neoepitopes formed when lipid peroxidation occurs. Surprisingly, innate immune recognition of these oxidation-specific epitopes is also prominent, and various macrophage scavenger receptors bind to epitopes of OxLDL (5-7). In addition, we previously found

Conflict of interest: Joseph L. Witztum is named as inventor in patents and patent applications from the UCSD for the potential commercial use of antibodies to oxidized LDL

Nonstandard abbreviations used: AP, alkaline phosphatase; CuOx-LDL, copper sulfate-oxidized LDL; ELISpot, enzyme-linked immunospot; FACS, fluorescence-activated cell sorting; 4-HNE-LDL, 4-hydroxynonenal-modified LDL; 4-HNE-MSA, 4-hydroxynonenal-modified mouse serum albumin; ISC, IgM-secreting cell; MAA-BSA, malondialdehyde-acetaldehyde-modified BSA; MAA-MSA, malondialdehyde-acetaldehyde-modified mouse serum albumin; MDA-LDL, malondialdehyde-modified LDL; NAb, natural Ab; OxLDL, oxidized LDL; OxPL, oxidized phospholipid; PAMP, pathogen-associated molecular pattern; PC, phosphocholine; PC-BSA, PC-conjugated BSA; PC-KLH, PC-conjugated keyhole limpet hemocyanin; PEC, peritoneal exudate cell; PRR, pattern recognition receptor; SPF, specific pathogen-free.

Citation for this article: J. Clin. Invest. 119:1335-1349 (2009). doi:10.1172/JCI36800. that innate natural Abs (NAbs) bind to oxidized phospholipids (OxPLs) of OxLDL. For example, cholesterol-fed apoE-deficient mice have very high IgM titers to OxLDL, which enabled cloning of IgM-secreting hybridomas from the spleens of these mice with specificity for OxLDL (8). A large number of these bound to both the lipid and apoB moieties of OxLDL, and specifically to the phosphocholine (PC) headgroup of OxPL, such as 1-palmitoyl-2-(5'-oxovaleroyl)-sn-glycero-3-phosphocholine (POVPC), present as either a lipid or as an adduct bound to protein via the $\varepsilon$-amino group of lysine. They did not bind to the PC of native phospholipids (9). Importantly, these antibodies, as represented by the prototypic antibody E06, inhibited the uptake of OxLDL by macrophage scavenger receptors CD36 and SR-BI $(5,6,10)$, as did POVPC linked to BSA or a peptide. This demonstrates that the PC moiety of OxPL is a ligand for macrophage scavenger receptors, which are innate, cellular pattern recognition receptors (PRRs).

Because all of these cloned autoantibodies were IgM Abs, which are thought in large part to represent NAbs in uninfected mice (11), we sequenced the complementarity-determining regions (CDRs) determining their antigen-binding sites, which revealed them all to be genetically identical to a well-characterized B-1 cell clone, T15, described more than 30 years ago (12). T15 NAbs bind to PC covalently linked to the cell wall polysaccharide (C-PS) of pathogens and provide optimal protection to mice from lethal infection with Streptococcus pneumoniae (13). Furthermore, immunization of cholesterol-fed $\mathrm{Ldll}^{-}$- mice with heat-killed S. pneumoniae led to a nearly exclusive expansion of E06/T15 NAbs and atheroprotection (14).

Because NAbs are postulated to be conserved by natural selection, it was not apparent what the selecting agent might be, as 
A

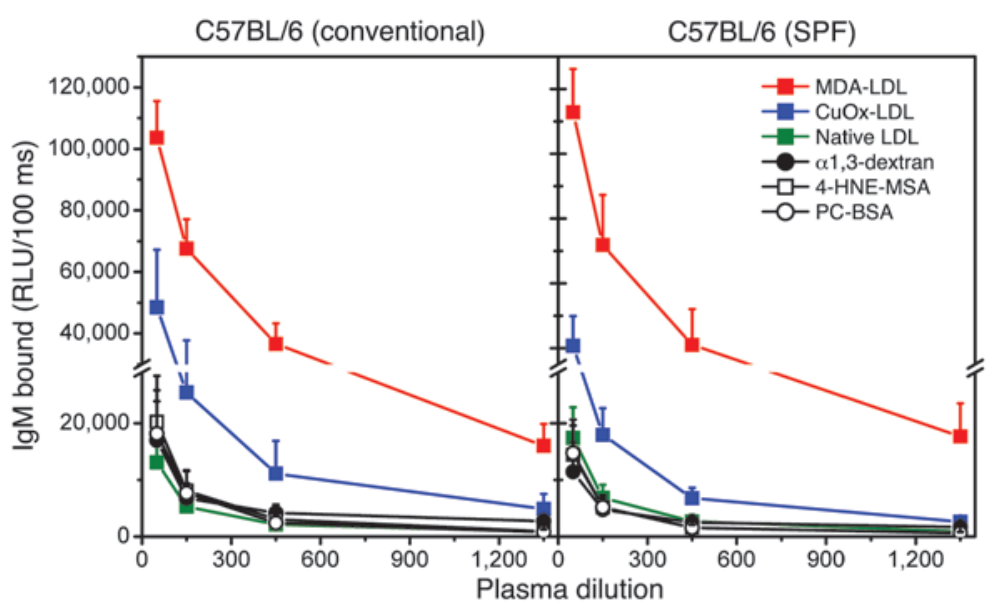

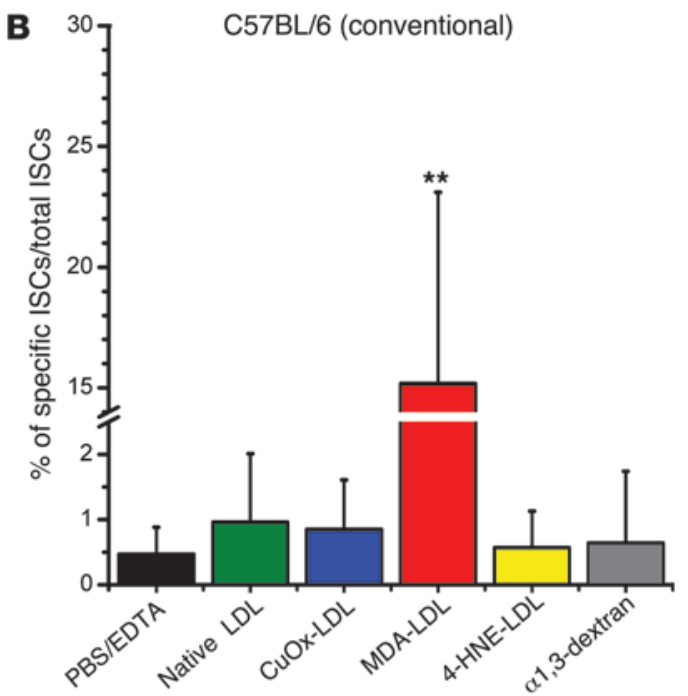

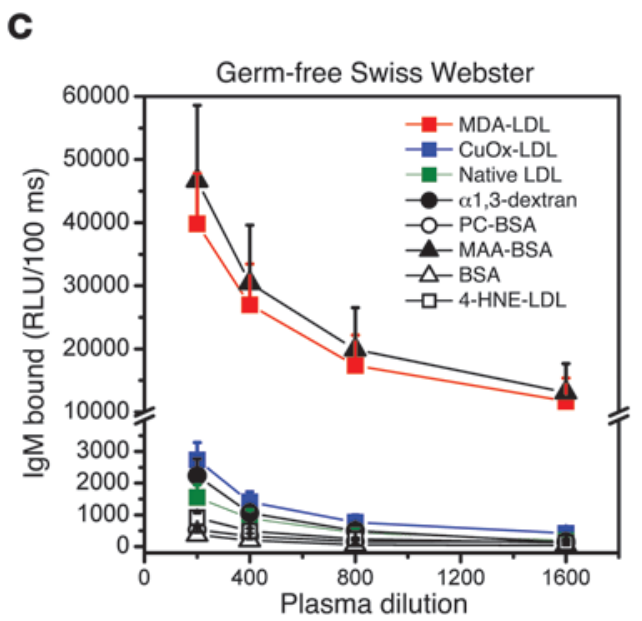

D

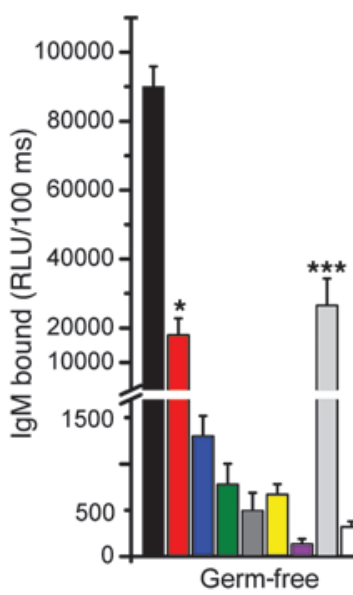

Swiss Webster

Total $\lg M$

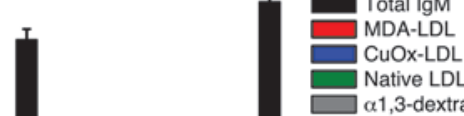

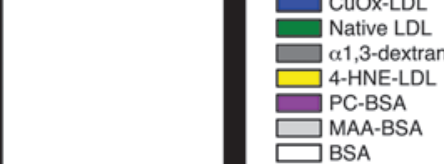
BSA

Figure 1

IgM Abs to oxidation-specific antigens are present in germ-free and conventional mice. (A) Conventional and SPF C57BL/6 mice have similar IgM titers to oxidation-specific antigens. Plasma from 11-week-old female conventionally raised $(n=4)$ and SPF $(n=4)$ C57BL/6 mice were tested by ELISA. Values are mean and SEM. (B) MDA-LDL-specific ISCs are dominant in the spleens of conventionally raised C57BL/6 mice. Splenocytes from conventionally raised 12-week-old female C57BL/6 mice $(n=4)$ were tested by ELISpot assay for frequencies of ISCs as described in Methods. Values represent the number of ISCs to indicated antigen as a percentage of total ISCs (mean and SD). Data are from 1 experiment representative of $3 .{ }^{* \star} P<0.01$ compared with all other antigens (1-way ANOVA with Tukey-Kramer multiple comparison test). (C) Binding curves of plasma IgM from germ-free Swiss-Webster mice to indicated antigens. Plasma samples were from 14- to 16-week old female and male mice $(n=9)$. Values are mean and SEM. (D) Titers of IgM Abs to oxidation-specific epitopes are present in conventional and germ-free Swiss Webster mice. Serum from 14- to 16-week-old female and male conventionally raised $(n=7)$, conventionalized (germ-free colonized with bacterial flora) $(n=11)$, and germ-free $(n=9)$ mice were diluted 1:400 and tested for binding to the indicated antigens. Values are mean and SEM. ${ }^{\star} P<0.05,{ }^{* \star} P<0.01,{ }^{* \star} P<0.002$ compared with $\alpha 1$,3-dextran (1-way ANOVA with Tukey-Kramer multiple comparison test).

oxidation of LDL and atherosclerosis per se should not exert any positive selective pressure. We postulated that apoptotic cells, similar to OxLDL, would also display oxidation-specific epitopes on their surface, as cells undergoing programmed cell death are known to undergo enhanced oxidative processes $(15,16)$ and if not promptly cleared are likely to be proinflammatory $(17,18)$. Indeed, using mass spectroscopy, we demonstrated that apoptotic cells contained an enhanced content of OxPL in their membranes and that E06 bound prominently to their cell surface, consistent with this hypothesis $(18,19)$. We also demonstrated that C-reactive protein (CRP), an innate acute-phase protein, recognized the same
PC moiety on OxLDL and apoptotic cells (20). These data strongly suggest that the PC moiety of OxPL, apoptotic cells, and the cell wall of bacteria constitute a pathogen-associated molecular pattern (PAMP) recognized by multiple arcs of innate immunity and that each could exert positive selective pressure.

A variety of such oxidation-specific epitopes, besides PC of OxPL, are likely to occur in abundance not only on apoptotic cells, but on shed microparticles, and in general on membranes and even bacteria during inflammatory responses. We postulated that they might constitute a previously unrecognized but important class of PAMPs and in turn would be a major target of innate NAbs. In 
A

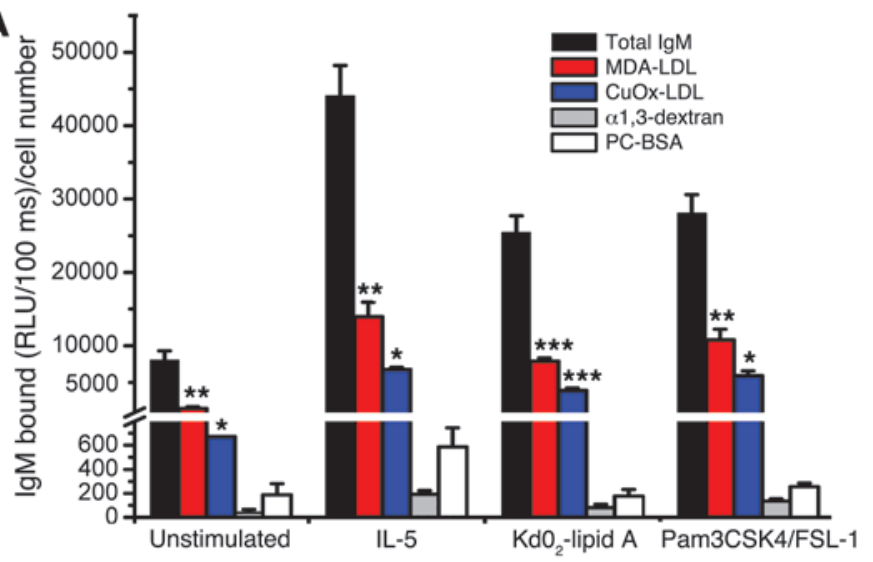

B

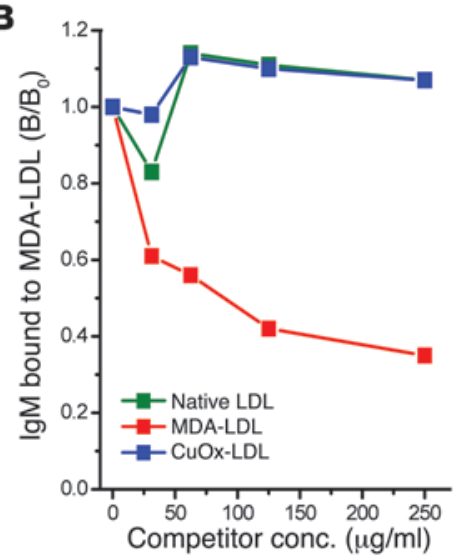

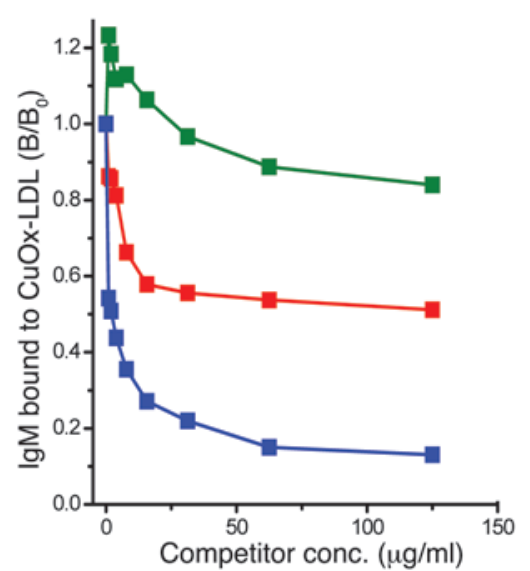

Figure 2

In vitro stimulation of B-1 cells induces increased natural $\operatorname{lgM} \mathrm{Ab}$ titers to oxidation-specific antigens. (A) Purified B-1 cells were cultured in 24-well plates in triplicate at a cell density of $1 \times 10^{6}$ cells per well in $500 \mu \mathrm{l}$ culture medium. Cells were stimulated with IL-5 $(50 \mathrm{ng} / \mathrm{ml})$, $\mathrm{KdO}_{2}$-Lipid A (100 $\mathrm{ng} / \mathrm{ml}$ ), or TLR2 agonists (a combination of Pam3CSK4 [300 ng/ml] and FSL-1 [1 $\mu \mathrm{g} / \mathrm{ml}]$ ) and incubated at $37^{\circ} \mathrm{C}$ for 7 days. Control B-1 cells were cultured in medium alone. Cell culture supernatants were harvested after 7 days and IgM Ab titers analyzed by ELISA at 1:45 dilution. Results were normalized to cell number recovered after 7 days. Values are mean and SEM. Data are from 1 experiment representative of 3. ${ }^{*} P<0.05,{ }^{* *} P<0.01,{ }^{* * *} P<0.002$ compared with $\alpha 1,3-$ dextran (repeated-measures ANOVA with Tukey-Kramer multiple comparison test). (B) Natural IgM Abs produced in vitro show specificity to MDA-LDL and CuOx-LDL. For competition immunoassay, supernatants from purified B-1 cell cultures stimulated with $\mathrm{KdO}_{2}$-Lipid A (100 ng/ $\mathrm{ml})$ or IL-5 $(50 \mathrm{ng} / \mathrm{ml})$ were diluted to 1:20 and incubated in the presence of the indicated concentrations of competitors (Competitor conc.) overnight. After incubation, IgM binding to MDA-LDL and CuOx-LDL was tested by ELISA. Data are the mean of triplicate determinations, expressed as ratio of IgM binding to MDA-LDL or CuOx$\mathrm{LDL}$ in the presence or absence of competitor $\left(B / \mathrm{B}_{0}\right)$. Data are from 1 experiment representative of 3. this article, we provide multiple lines of evidence suggesting that oxidation-specific epitopes are a dominant target of innate NAbs in both mice and humans.

\section{Results}

IgM Abs against oxidation-specific epitopes are present in normal and germfree mice. To characterize the murine humoral IgM responses to defined oxidation-specific epitopes, we assessed specific IgM titers in plasma of naive, nonatherosclerotic C57BL/6 mice. As previously observed (8), prominent IgM titers to oxidation-specific epitopes, such as OxLDL $(>1: 1,350)$ and malondialdehyde-modified LDL (MDA-LDL) (>>1:1,350), and to 4-hydroxynonenal-modified mouse serum albumin (4-HNE-MSA) and PC-conjugated BSA (PC-BSA; 1:1,350), can be detected even in normal, conventionally housed mice, whereas IgM titers to "native LDL" are minimal or undetectable (Figure 1A) (see comment on apparent binding to native LDL below under the subhead IgM binding to native $L D L$ ). Among these, the IgM responses to MDA modifications were consistently found to be the most robust, and the titers were many fold higher than the titer $(1: 1,350)$ to the prototypic B- 1 cell antigen $\alpha 1,3$-dextran. In addition, when comparing the plasma IgM titers to those in age-matched mice bred under specific pathogen-free (SPF) conditions, a similar response pattern was observed (Figure $1 \mathrm{~A})$, suggesting that the basal titers of these IgM Abs are largely independent of noncommensal exposure to microbial pathogens. Moreover, we found that oxidation-specific IgM levels were also present in $\mathrm{T}$ cell receptor-deficient $\left(\mathrm{Tcra}^{-/}\right)$mice - although slightly lower - indicating that in large part these responses do not require T cells (see Supplemental Figure 1; supplemental material available online with this article; doi:10.1172/JCI36800DS1).

In mice, IgM Abs are in large part derived from Ab-secreting cells in the spleen (21). Using enzyme-linked immunospot (ELISpot) analysis, we tested the frequencies of IgM-secreting cells (ISCs) against candidate oxidation-specific epitopes in the spleens of conventionally housed C57BL/6 mice. ISCs with specificity for oxidation-specific epitopes were equally prominent in the spleen, as was observed for the IgM in plasma, with up to $15 \%$ of all ISCs having specificity for MDA-LDL (Figure 1B).

Our data suggest that IgM titers to an array of oxidation-specific epitopes may in fact represent IgM generated even in the absence of response-eliciting antigen exposure. In confirmation of this, we demonstrated robust IgM titers to oxidation-specific epitopes in the serum of "germ-free mice," which are completely free of gut bacteria (Figure 1C). In particular, IgM titers to MDALDL $(>1: 1,600)$ and MAA-BSA $(>1: 1,600)$ were the most prominent among all IgM titers measured: MAA (malondialdehyde-acetaldehyde adduct) is a specific and prominent chemical moiety generated from 2 MDA and 1 acetaldehyde molecules reacting with the $\varepsilon$-amino group of lysine to form an adduct; in this case forming adducts with BSA. Titers to OxLDL (>1:1,250) and 4-hydroxynonenal-modified LDL (4-HNE-LDL; 1:800) were also much higher than those to $\alpha 1,3$-dextran (1:400), while titers to PC-BSA, which shares molecular identity to the PC of OxPL (as found in OxLDL), were approximately 1:400.

Furthermore, reconstitution of germ-free mice with gut bacteria ("conventionalized mice") for only 2 weeks led to increases in 
A
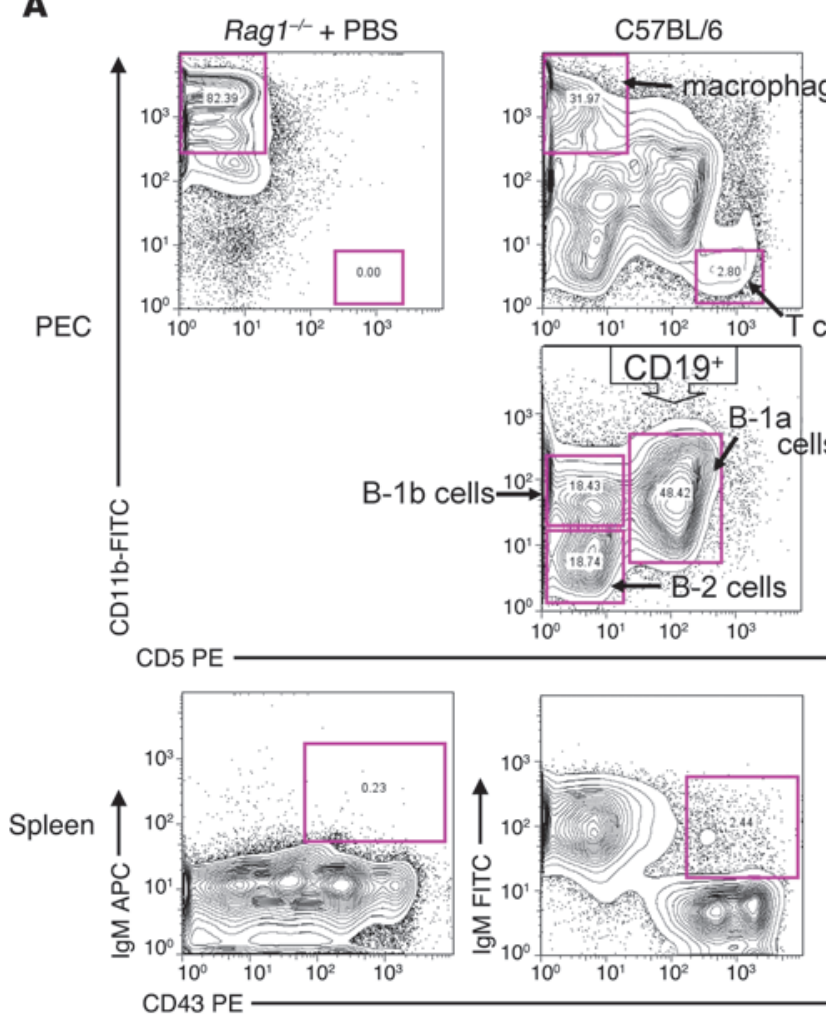

B
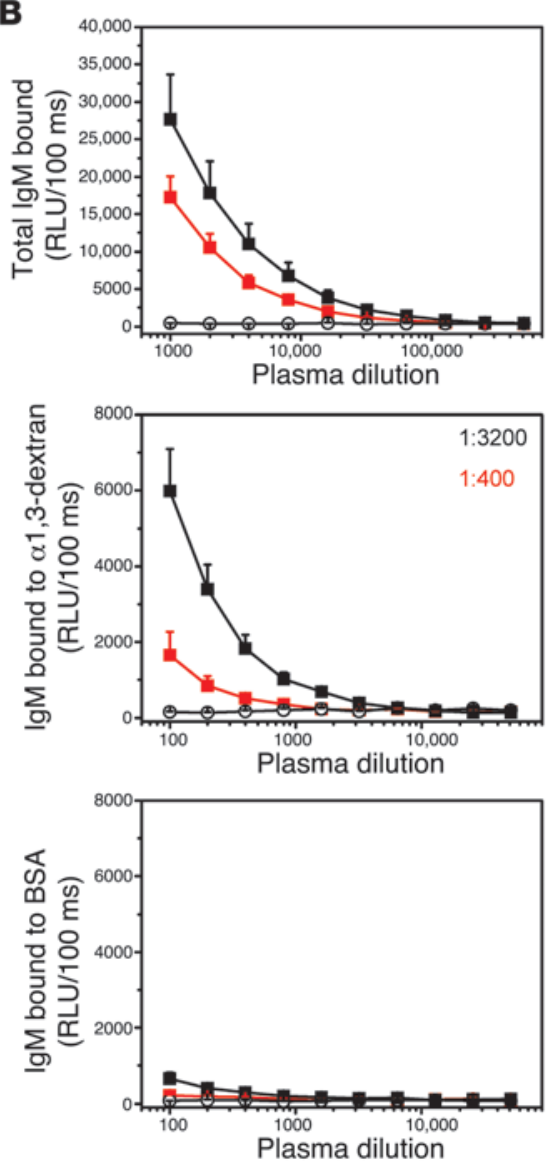
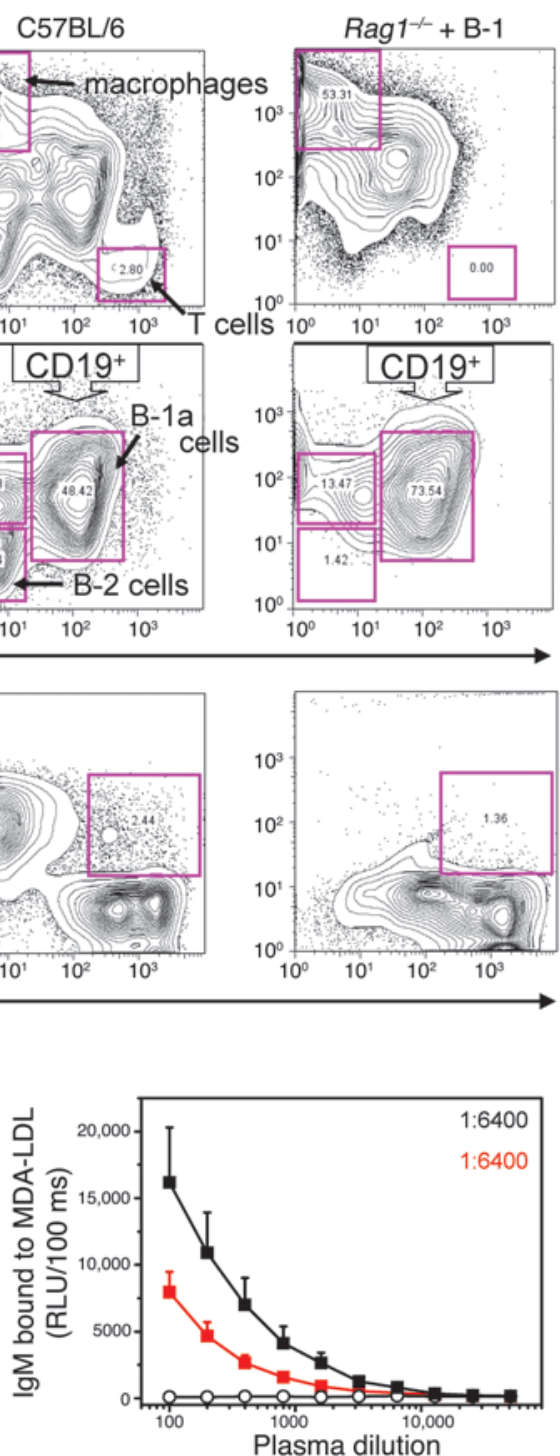

C
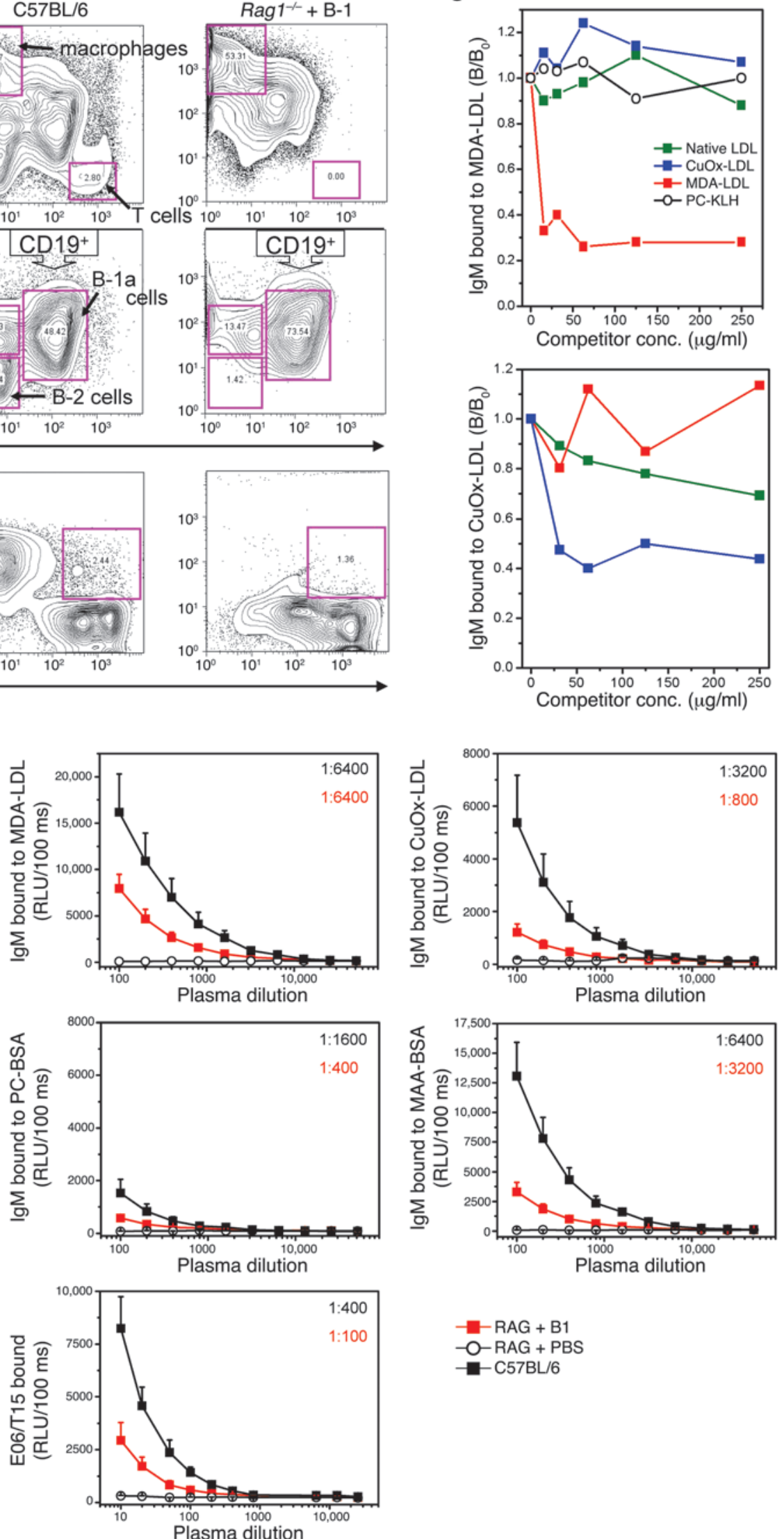

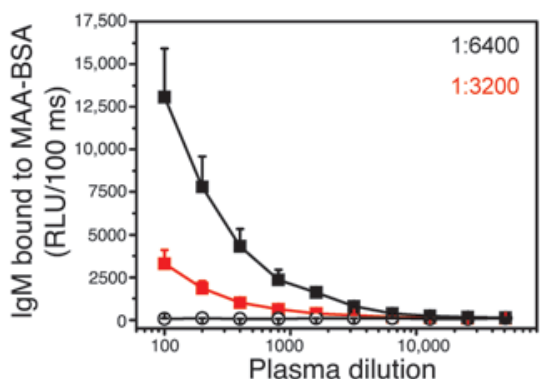

- RAG + B1

$-\mathrm{O}-\mathrm{RAG}+\mathrm{PBS}$

$-\mathrm{C} 57 \mathrm{BL} / 6$ 


\section{Figure 3}

Characterization of Rag $^{-1-}$ recipients adoptively transferred with B-1 cells. (A) Adoptive transfer of B-1 cells into Rag 1-/- mice replenishes B-1 cell population. Rag $1^{-/-}$mice were injected with PBS (Rag $1^{-1-}+$ PBS) or with B-1 cells (Rag $\left.1^{-1-}+\mathrm{B}-1\right)$. Rag $1^{-/}+$PBS: Lymphocyte populations were absent in the peritoneal cavity (PEC, left). B-1 cells $\left(\operatorname{lgM}{ }^{+} \mathrm{CD}^{2} 3^{+}\right)$were also absent from the spleen (Spleen, left). C57BL/6: Peritoneal macrophages (CD11 $\mathrm{b}^{\text {hi }}$ CD5-) and T cells (CD11 b-CD5 ${ }^{\text {hi }}$ ) were intact (PEC, upper middle). $\mathrm{B}$ cells could be divided into $\mathrm{B}-1 \mathrm{a}$ (CD19+CD11 bint CD5 $\left.{ }^{\text {int }}\right), B-1 b\left(C D 19+C D 11 b^{\text {int }}\right.$ CD5-), and B-2 cells (CD19+CD11b-CD5-) (PEC, lower middle). In the spleen, B-1 cells were about $2.4 \%$ of total splenocytes (Spleen, middle). Rag $1^{-/}+\mathrm{B}-1$ : $\mathrm{B}-1$ cell populations were reconstituted in the peritoneal cavity (PEC, lower right) and spleen (Spleen, right), without B-2 cell or T cell contamination (PEC, right). (B) IgM Abs to oxidation-specific epitopes are present in the plasma of $\mathrm{B}-1$ reconstituted Rag $1^{-/-}$mice. Plasma collected after 15 weeks from Rag 1-/- + B-1 $(n=8)$ or Rag 1-/- + PBS $(n=6)$ and age-matched C57BL/6 mice $(n=7)$ were tested. Data shown are from 1 transfer experiment representative of 6 . Values are mean and SEM. Numbers in the upper-right corner represent the IgM titer to each antigen. (C) Natural IgM Abs produced in vivo show specificity to MDA-LDL and CuOx-LDL. Data are the mean of triplicate determinations, expressed as the ratio of IgM binding to MDA-LDL or $\mathrm{CuOx}-\mathrm{LDL}$ in the presence or absence of competitor $\left(\mathrm{B} / \mathrm{B}_{0}\right)$. Data are from 1 experiment representative of 3 .

many - but not all - of the oxidation-specific IgM levels (Figure 1D), strongly suggesting molecular mimicry between many endogenous oxidation-specific epitopes and gut bacterial epitopes. While IgM responses to PC and 4-HNE, as well as to $\alpha 1,3$-dextran, were increased (more than 2-fold) in these mice, the MDAspecific responses were found to be similar to those in germ-free mice. Moreover, similar specific IgM responses were measured in age-matched conventionally raised mice of the same genetic background (Figure 1D). Note again that oxidation-specific IgM Abs constitute a major fraction of total IgM Abs, which were not different among wild-type, germ-free, or colonized mice (Figure 1D).

IgM binding to native $L D L$. In these studies, we tested IgM binding to antigens coated on microtiter wells using standard solid-phase ELISA techniques (22). In some experiments, we saw low to modest levels of IgM binding to native LDL, which appeared to vary with different LDL preparations. However, in all cases, the binding to plated native LDL could not be competed by the same preparation of native LDL in solution (data not shown), suggesting that the LDL became modified in some way during the plating process. It should be emphasized that in competition immunoassays, we never observed native LDL competing for binding to any of the modified LDL preparations (e.g., as shown in Figure 2B, Figure $3 \mathrm{~A}$, and Figure 4A).

B-1 cells secrete IgM NAbs against oxidation-specific epitopes in vitro. To directly demonstrate that oxidation-specific IgM Abs are derived from innate B- 1 cells, we isolated B- 1 cells (both $\mathrm{CD}^{+} \mathrm{B}-1 \mathrm{a}$ and $\mathrm{CD5}^{-} \mathrm{B}-1 \mathrm{~b}$ ) from naive mice by fluorescence-activated cell sorting (FACS), stimulated them in vitro with various stimuli, and tested the culture supernatants for specific IgM Abs. IL-5, a TLR4 ligand $\left(\mathrm{KdO}_{2}\right.$-Lipid A), as well as a combination of TLR2 ligands (FSL-1 and Pam3CSK4) all induced B-1 cells to secrete IgM against MDALDL, OxLDL (Figure 2A), and 4-HNE-LDL (data not shown); but also against the prototypic B-1 cell antigen $\alpha 1,3$-dextran (Figure $2 \mathrm{~A}$ ). Utilizing B-1 cells from $M y d 88^{-/-}$mice, we observed that the responses to both TLR4 and TLR2 agonists were in large part MyD88 depen- dent (data not shown). Interestingly, the basal secretion of IgM to OxLDL and MDA-LDL was strikingly more prominent than that of IgM against $\alpha 1,3$-dextran (Figure $2 \mathrm{~A}$ ), and in response to stimulation with the TLR agonists, the oxidation-specific IgM increased to a greater extent than did the total IgM, or the IgM to $\alpha 1,3$-dextran, for example (Supplemental Figure 2). Basal titers to PC-BSA were relatively low and increased only in response to IL-5. PC is an epitope for some OxLDL-specific IgM, but not all.

By analogy to the robust anti-MDA responses in vivo, MDA-specific IgM Abs were the dominant set of IgM Abs secreted by B-1 cells in vitro. Although we tested only a narrowly selected set of antigens, the anti-MDA Abs constituted up to $30 \%$ of total IgM secreted. These Abs were highly specific for MDA modifications, as only MDA-LDL, but neither OxLDL nor native LDL, competed for the binding (Figure 2B), while IgM Abs bound to plated OxLDL were competed by both OxLDL and MDA-LDL. We also calculated the binding avidities of the IgM in the supernatants for MDA-LDL and OxLDL using the Klotz method (23). The calculated $K_{d}$ s for MDA-LDL and OxLDL were $1.46 \times 10^{-7} \mathrm{~mol} / \mathrm{l}$ and $4.03 \times 10^{-9} \mathrm{~mol} / \mathrm{l}$, respectively, similar to values we previously determined for IgM in plasma of mice immunized against these epitopes (18).

$B-1$ cells secrete IgM NAbs against oxidation-specific epitopes in vivo. To test whether innate B-1 cells can also secrete oxidation-specific IgM in vivo, peritoneal B- 1 cells from naive C57BL/ 6 mice were adoptively transferred into the peritoneum of $\mathrm{Ragl}^{-/-}$recipient mice, which lack functional $\mathrm{B}$ and $\mathrm{T}$ cells. This led to the selective reconstitution of only B-1 cells in the peritoneum of Rag $1^{-/-}$recipients (Figure 3A, top row), and both B-1a $\left(\mathrm{CD}^{+} \mathrm{CD} 19^{+} \mathrm{CD} 11 \mathrm{~b}^{+}\right)$ and $\mathrm{B}-1 \mathrm{~b}$ cells $\left(\mathrm{CD} 5^{-} \mathrm{CD} 19^{+} \mathrm{CD} 11 \mathrm{~b}^{+}\right.$) were detected (Figure $3 \mathrm{~A}$, middle row), whereas conventional B-2 cells and T cells were typically not found in recipient mice. The average percentages of $\mathrm{B}-1 \mathrm{a}$ and B-1b cells among total cells analyzed in the peritoneum of Rag1 $1^{-/-}$B-1 recipients were $11.0 \% \pm 2.6 \%$ and $8.5 \% \pm 1.0 \%$ respectively $(n=12)$, compared with $18.2 \% \pm 3.2 \%$ and $8.3 \% \pm 2.3 \%(n=4)$ in wild-type C57BL/ 6 mice.

The adoptive transfer also reconstituted the B- 1 cell population in the spleens of Rag $^{-/-}$B-1 recipients. B-1 cells (IgM- and CD43positive; Figure $3 \mathrm{~A}$, bottom row) constituted an average of about $1 \%$ of total splenocytes analyzed (data ranged from $0.4 \%$ to $1.4 \%$; $n=9$ ), while in wild-type C57BL/6 mice, B-1 cells averaged about $2.7 \%(n=3)$. Moreover, this reconstitution was also demonstrated by the number of splenic ISCs as measured by ELISpot: $60 \pm 15$ ISCs vs. $152 \pm 21$ ISCs per 200,000 splenocytes in Rag $1^{-/-} \mathrm{B}-1$ recipients $(n=9)$ and C57BL/6 mice $(n=7)$, respectively.

The B-1 cell-reconstituted Rag1-/- mice developed readily detectable plasma titers of IgM by the tenth week after transfer (Figure $3 \mathrm{~B}$ ), while $\mathrm{Rag}^{-1^{--}}$mice that received only PBS did not have any plasma IgM. In a series of 8 similar transfer experiments, the extent of reconstitution, as indicated by the levels of plasma IgM, varied and in part appeared to be related positively to the number of B-1 cells transferred and the time after transfer studied. Remarkably, in all of the transfers, the recipient mice exhibited robust IgM titers against oxidation-specific epitopes (Figure 3B), but not native LDL (data not shown), and the prevalence of oxidation-specific IgM appeared similar to that observed in naive wild-type mice. In Figure $3 \mathrm{~B}$, we show $\mathrm{Ab}$ binding dilution curves to oxidation-specific epitopes and other antigens for a typical transfer of approximately $99 \%$ pure B-1 cells. The titers ranged from 1:800 to $1: 6,400$ for oxidation-specific epitopes versus 1:400 for $\alpha 1,3$-dextran and PCBSA. Moreover, consistent with the fact that T15-idiotypic Abs are 

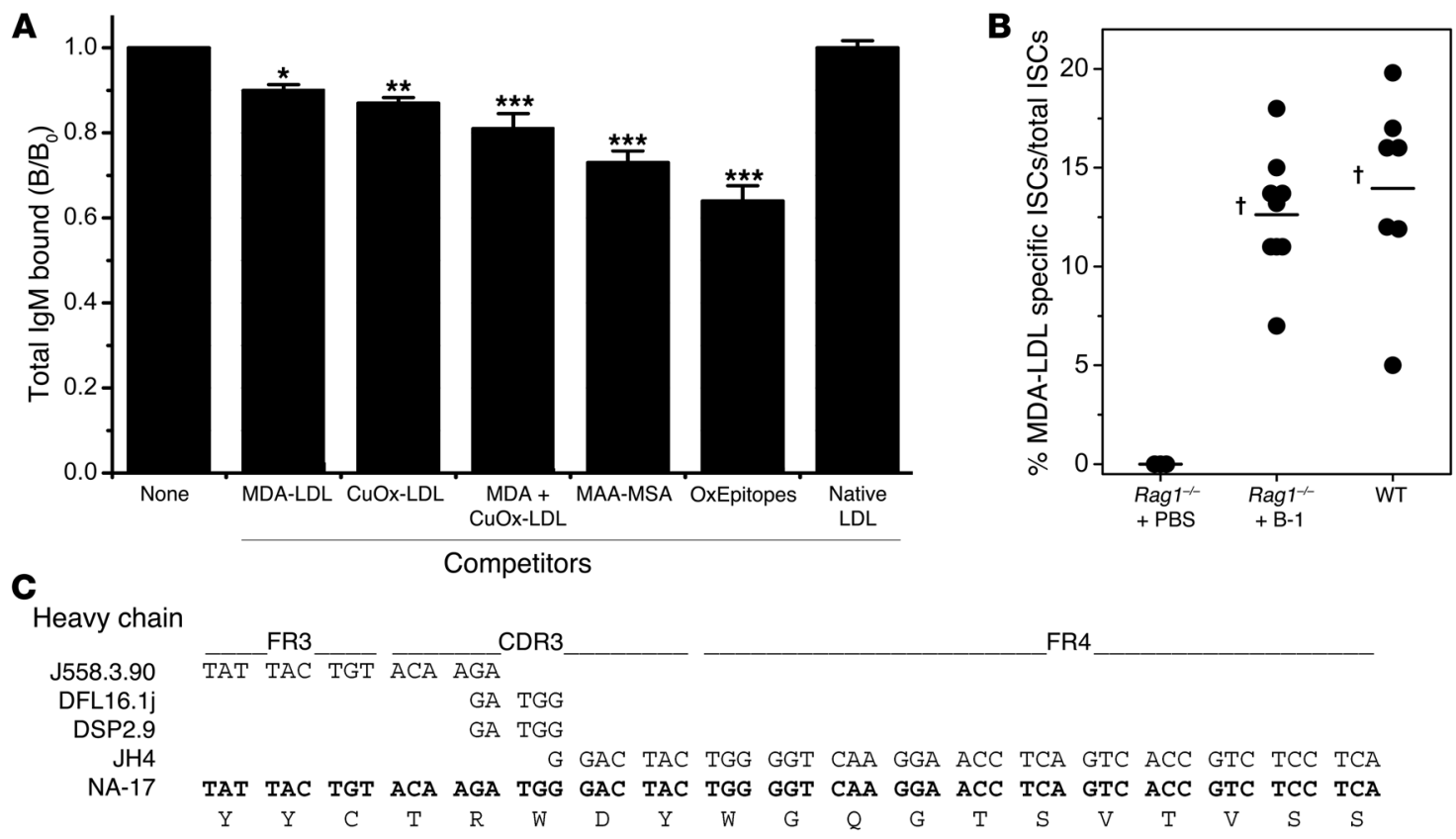

Light chain

\begin{tabular}{|c|c|c|c|c|c|c|c|c|c|c|c|c|c|c|c|c|c|c|c|c|c|c|c|c|}
\hline \multirow{3}{*}{$\begin{array}{r}\text { Kh4 } \\
\text { JH5 }\end{array}$} & \multicolumn{4}{|c|}{ FR3 } & \multicolumn{9}{|c|}{ CDR3 } & \multicolumn{11}{|c|}{ FR4 } \\
\hline & $\overline{C T}$ & TAT & $\overline{T A C}$ & $\overline{\mathrm{GT}}$ & $\overline{A A}$ & $\mathrm{CAG}$ & TGG & $\mathrm{AAT}$ & $\mathrm{AGT}$ & TAC & $\overline{C A}$ & $C$ & & & GGT & & GGG & $A C C$ & AAG & G & GAG & CTG & A & CGT \\
\hline & $\mathrm{ACT}$ & TAT & TAC & TGT & CAA & CAG & TGG & AAT & AGT & TAC & CCA & $\mathrm{CCC}$ & ACG & TTC & GGT & GCT & GGG & $\mathrm{ACC}$ & AAG & CTG & GAG & CTG & $\mathrm{AAA}$ & GGT \\
\hline & $\mathrm{T}$ & $Y$ & $\mathrm{Y}$ & $C$ & $Q$ & $Q$ & $\mathrm{~W}$ & $\mathrm{~N}$ & $S$ & $\mathrm{Y}$ & $\mathrm{P}$ & $\mathrm{P}$ & $\mathrm{T}$ & $F$ & G & A & G & $\mathrm{T}$ & $\mathrm{K}$ & $\mathrm{L}$ & $E$ & $\mathrm{~L}$ & $\mathrm{~K}$ & G \\
\hline
\end{tabular}

Figure 4

Oxidation-specific epitopes are dominant targets of NAbs. (A) Preabsorption of plasma from Rag 1-/- B-1 mice with oxidation-specific antigens shows that oxidation-specific epitopes (OxEpitopes) are dominant targets for NAbs. Plasmas from Rag $1^{-/-}+\mathrm{B}-1$ mice were preincubated in the absence or presence of the indicated antigens $(250 \mu \mathrm{g} / \mathrm{ml}$ total antigen) overnight and antigen-immune complexes pelleted by centrifugation. Total IgM levels were then tested by ELISA. ${ }^{*} P<0.05$, ${ }^{* \star} P<0.01$, ${ }^{* \star *} P<0.002$ compared with native LDL (ANOVA with Tukey-Kramer multiple comparisons test). Data are means (and SEM) from 5 separate experiments, each using 3-7 plasma samples obtained from 5 different transfer experiments, with each sample assayed in triplicate. (B) ELISpot assay of frequencies of MDA-LDL-specific ISCs in the spleens of wild-type C57BL/6, Rag 1 $1^{-1-}+\mathrm{B}-1$, and Rag $1^{-/-}+$PBS mice. Results are from individual mice, and data are from 3 separate B-1 cell transfer experiments. Horizontal bar represents the mean for the group. ${ }^{\dagger} P<0.002$ compared with Rag $1^{-/-}+$PBS (unpaired $t$ test). (C) B-1 cell-derived natural mAb NA-17. DNA sequences of VDJ splice sites of the $V_{H}$ and $V_{L}$ rearrangements expressed in NA-17 B-1 cell hybridoma and their relationship to the most homologous germline V, D, J gene segments. Sequence analysis of NA-17 $\mathrm{V}_{H}$ rearrangement did not reveal nucleotide variation to germline genes. Sequence analysis of $V_{L}$ rearrangement revealed 1 nucleotide insertion between $V_{L}$ and $J_{L}$ germline gene segments.

predominantly secreted by B-1 cells (24), we found that adoptive $\mathrm{B}-1$ cell transfer gave rise to E06/T15-idiotypic IgM in Rag $1^{-/-}$mice as well (Figure 3B, bottom middle panel).

Whereas the extent of plasma IgM measured at 10 weeks was generally lower in B-1 cell-reconstituted Rag $1^{-/-}$than in wild-type mice in all the experiments noted above, in which greater than $99 \%$ pure B-1 cells were transferred, in one experiment, in which a small contamination of $T$ cells inadvertently occurred (estimated to be $<3 \%$ of all peritoneal cells at sacrifice), the IgM levels actually equaled those of wild-type C57BL/6 mice (Supplemental Figure 3). Presumably, cotransferred $\mathrm{T}$ cells promoted IgM secretion in the recipient mice, possibly in part by secretion of cytokines such as IL-5, which, as we have previously shown, augments secretion of OxLDL-specific IgM by B-1 cells in a non-cognate manner (Figure 2A and ref. 25).

Again, oxidation-specific IgM Abs were a major fraction of the total IgM. Competition immunoassays with pooled plasma of recipient mice demonstrated high specificity of the MDA-specific IgM Abs, as neither OxLDL nor native LDL competed for binding to MDA-LDL (Figure 3C). We also calculated binding avidities for the
IgM in the B-1 cell-reconstituted Rag $1^{-/-}$plasma for oxidation-specific epitopes as described above. The apparent $K_{d}$ s for MDA-LDL and OxLDL were $9.9 \times 10^{-9}$ and $1.42 \times 10^{-7} \mathrm{~mol} / \mathrm{l}$ respectively. These values are similar to the $K_{d} \mathrm{~S}$ of $6.85 \times 10^{-8} \mathrm{~mol} / \mathrm{l}$ determined for the MDA-LDL-specific natural mAb NA-17, cloned from the spleen of a B-1 cell reconstituted Rag $1^{-/-}$mice as described below. Only very low titers of IgG against oxidation-specific epitopes (or any other antigen) were found in the plasma of recipient mice, and these Abs were predominantly of the IgG3 isotype, which are known to be secreted by B-1 cells in a T cell-independent fashion (26) (data not shown).

Oxidation-specific epitopes are dominant targets of natural IgM Abs. To directly address the extent to which oxidation-specific IgM Abs contribute to the total IgM NAb pool, we performed absorption studies using pooled plasma from $\mathrm{Rag} 1^{-/-}$mice reconstituted with B-1 cells. In these experiments, specific IgM Abs were absorbed from plasma with selected oxidation-specific model antigens and the amount of remaining IgM measured. Strikingly, MDA-LDL as well as OxLDL (containing a variety of oxidation-specific epitopes) absorbed out approximately $10 \%$ of all IgM, while native LDL did 

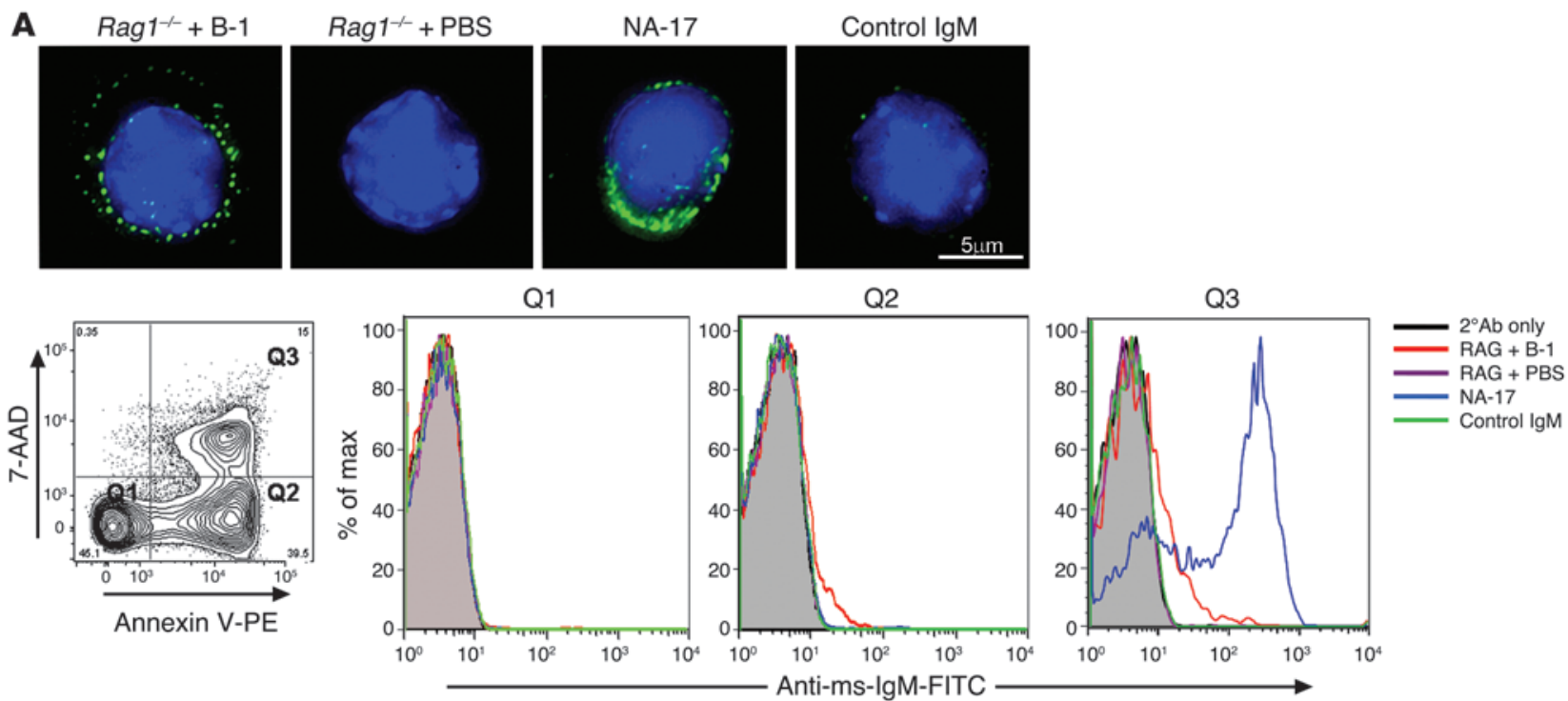

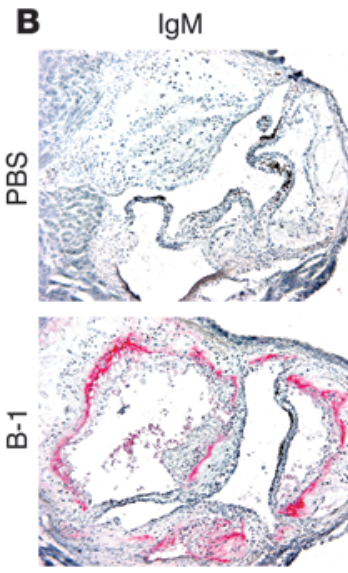

C

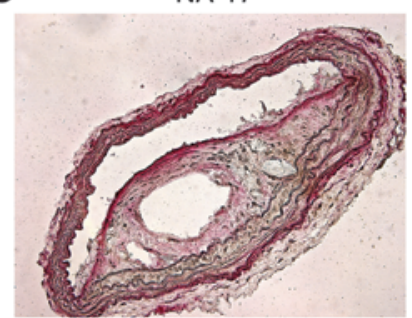

$\lg G$

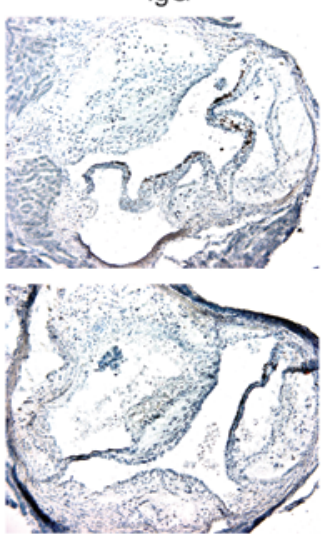

EN-2
D

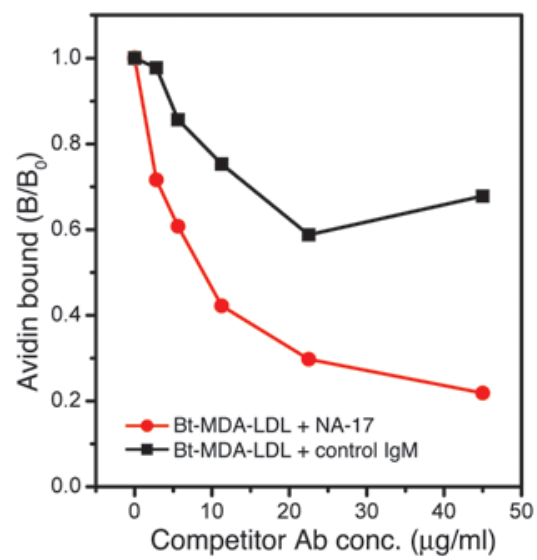

\section{Figure 5}

Natural IgM Abs recognize oxidation-specific epitopes present on apoptotic cells and atherosclerotic lesions (A) Natural IgM Abs bind to apoptotic thymocytes but not normal thymocytes. Apoptotic thymocytes from C57BL/6 mice were incubated with plasma from Rag $1^{-/-}+\mathrm{B}-1$ or Rag $1^{-/-}+$ PBS mice at 1:10 dilution, NA-17 at $2.5 \mu \mathrm{g} / \mathrm{ml}$, or control lgM at $5 \mu \mathrm{g} / \mathrm{ml}$. Top row: Deconvolution microscopy shows that NAbs in Rag $1^{-/-}+\mathrm{B}-1$ as well as NA-17 bound to apoptotic thymocytes. Bottom row: None of the IgM bound to normal thymocytes (quadrant 1 [Q1). NAbs in plasma from Rag $1^{-1-}+$ B-1 bound to both early (Q2) and late apoptotic thymocytes (Q3), while NA-17 bound prominently to late apoptotic cells (Q3). Scale bar: $5 \mu \mathrm{m} .2^{\circ} \mathrm{Ab}$, secondary Ab; Anti-ms-IgM-FITC, FITC-labeled anti-mouse IgM. (B) Natural lgM Abs are present in atherosclerotic lesions. Endogenous IgM Abs were detected in aortic sections from cholesterol-fed B-1 cell-reconstituted Ldl $r^{-1-}$ Rag $1^{-/-}$mice (bottom row), but not in PBS-injected $L d \mathrm{lr} \mathrm{r}^{-1-}$ Rag $1^{-1-}$ mice (top row). Sections were also stained with MDA2 $(5 \mu \mathrm{g} / \mathrm{ml})$ for the presence of MDA epitopes. Red indicates positive staining. Original magnification, $\times 160$. (C) NA-17 recognizes oxidation-specific epitopes present in atherosclerotic lesions. Sections of the brachiocephalic artery from cholesterol-fed Ldl/ ${ }^{-1}$ Rag $1^{-1-}$ mice were stained with NA-17 (0.85 $\left.\mu \mathrm{g} / \mathrm{ml}\right)$ or a control natural IgM Ab, EN-2 $(1.6 \mu \mathrm{g} / \mathrm{ml})$. Original magnification, $\times 200$. (D) NA-17 inhibits MDA-LDL binding to macrophages. Increasing concentrations of NA-17 or control IgM were added with a fixed amount of biotinylated MDA-LDL (Bt-MDA-LDL; $2 \mu \mathrm{g} / \mathrm{ml}$ ) to macrophages. Data are the average of 2 experiments, expressed as the ratio of biotinylated MDA-LDL binding to macrophages in the presence or absence of $\operatorname{lgM}\left(B / B_{0}\right)$. 

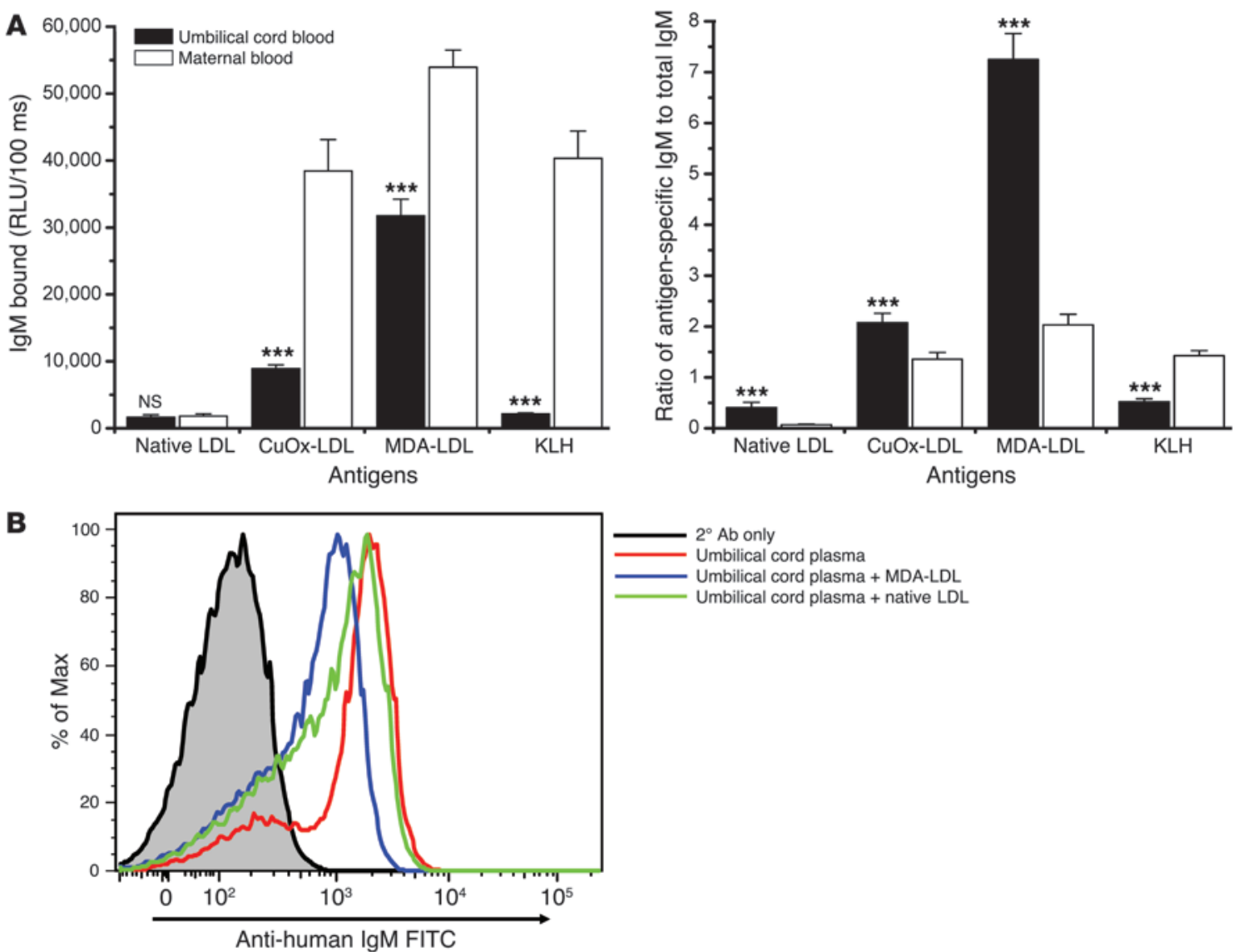

Figure 6

Human umbilical cord blood contains natural IgM Abs against oxidation-specific epitopes. (A) Left: Plasma titers of IgM in maternal and umbilical cord plasma to native LDL, KLH, and oxidation-specific antigens measured by ELISA. Right: Data are plotted as ratio of antigen-specific IgM to total IgM. ${ }^{* \star *} P<0.002$ compared with maternal blood (Wilcoxon matched-pairs test and paired $t$ test). Data shown are from 10 paired maternalinfant samples, and each sample was assayed in triplicate. Values are mean and SEM. (B) Umbilical cord IgM binds to apoptotic cells in part via binding to MDA. Apoptotic Jurkat cells, induced by UV exposure, were incubated with representative umbilical cord plasma (1:50 dilution) in the absence and presence of MDA-LDL and native LDL $(1 \mathrm{mg} / \mathrm{ml})$. Abs bound were detected by FITC-conjugated anti-human IgM. Umbilical cord IgM binding to apoptotic Jurkat cells (median fluorescence intensity [MFI], 1,917) was inhibited 45\% by MDA-LDL (MFI, 1,047) while minimally affected by native LDL (MFI, 1,514).

not (Figure 4A). Furthermore, the MDA-specific MAA epitope, conjugated to mouse serum albumin (MAA-MSA), was capable of absorbing up to $25 \%$ of all IgM. Moreover, a combination of all model antigens removed $35 \%$ of the NAbs in these plasmas (Figure 4A). Thus, a surprisingly large percentage of B-1 cell-derived NAbs are directed against various oxidation-specific epitopes.

The prominent representation of oxidation-specific IgM in plasma was also reflected by the frequency of MDA-specific ISCs in the spleens of reconstituted Rag $1^{-/-}$mice. As expected, no ISCs were detected in the spleens of mice injected with PBS (Figure 4B). In contrast, in B-1 cell-reconstituted mice, approximately $12 \%$ of all ISCs were found to have specificity for MDA-LDL (Figure 4B). Interestingly, the frequency of MDA-LDL-specific ISCs in spleens of wild-type mice was found to be similarly high (Figure 4B), demonstrating that a large percentage of all splenic ISCs have specificity for MDA modifications, and a majority of these ISCs are likely derived from B-1 cells.

We further corroborated this notion by the characterization of mAbs derived from hybridomas prepared from the spleens of B-1 cell-reconstituted Rag $1^{-/-}$mice. From 2 separate fusions, we observed that $20 \%-30 \%$ of all IgM-secreting hybridomas had reactivity for MDA-LDL (data not shown). For example, the DNA sequence of VDJ splice sites of the $V_{H}$ and $V_{L}$ rearrangements expressed in one cloned MDA-specific hybridoma (NA-17) displayed complete germline gene usage of the $\mathrm{V}_{\mathrm{H}}$ rearrangement and only 1 nucleotide insertion (C) at the splice site of the $V_{L}$ and $\mathrm{J}_{\mathrm{L}}$ germline gene segments (Figure $4 \mathrm{C}$; the complete sequence is presented in Supplemental Figure 4).

$B-1$ cell-derived natural IgM Abs recognize oxidation-specific epitopes on apoptotic cells and in atherosclerotic lesions. Oxidation-specific epitopes are ubiquitously present in inflammatory settings and are present on apoptotic cells $(19,27)$. As shown in Figure 5A, plasma IgM from B-1 cell-reconstituted Rag1 $1^{-/-}$mice recognized surface epitopes on apoptotic cells, as demonstrated by immunocytochemistry. Similarly, the MDA-LDL-specific NAb NA-17 strongly stained apoptotic cells. Neither plasma from PBS-injected Rag $1^{-/-}$mice nor a keyhole limpet hemocyanin-specific (KLH-specific) control IgM bound apoptotic cells. 

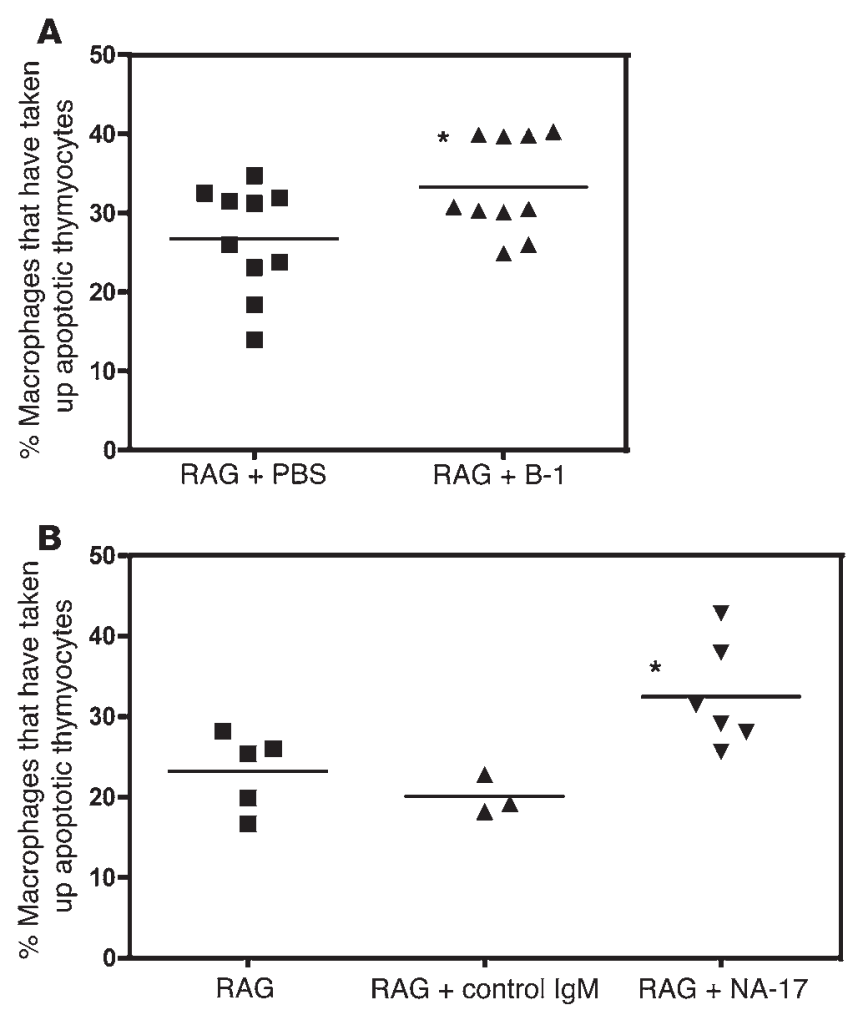

We also tested IgM binding to apoptotic cells by flow cytometry in relation to measures of cellular apoptosis (Figure 5A). Plasma IgM from reconstituted $\operatorname{Rag} 1^{-/-}$mice bound to apoptotic cells with early (quadrant 2 [Q2]) and late (Q3) stages of apoptosis, but not viable cells (Q1). Monoclonal NA-17 stained almost the entire population of late apoptotic cells. To further test whether these IgM NAbs recognize their cognate epitopes in vivo, we transferred B-1 cells into $\mathrm{Ldlr}^{-1-} \mathrm{Rag}^{-1-}$ mice that had been fed a high-cholesterol diet for 10 weeks to induce atherosclerotic lesions, which accumulate apoptotic cells and $\operatorname{OxLDL}(28,29)$. The mice were maintained on the atherogenic diet for an additional 6 weeks and then sacrificed, and atherosclerotic lesions were assessed for the deposition of endogenous Abs (Figure 5B). As expected, neither IgM nor IgG Abs were present in lesions of $\mathrm{Ldlr}^{-/} \mathrm{Rag} 1^{-/-}$mice injected with PBS alone (Figure 5B, top row). In contrast, IgM accumulated in lesions of B-1 cell-reconstituted mice, at sites likely reflecting the edges of lesions at the time of engraftment of the transferred B-1 cells (Figure 5B, bottom row). In part, these IgM Abs were binding to endogenous MDA epitopes in the lesions, which were found in comparable (but not identical) sites, as demonstrated by immunostaining of adjacent sections using MDA2, an MDAspecific murine monoclonal IgG we previously cloned (30) (Figure 5B). Presumably MDA epitopes bound by endogenous IgM would also not be available to bind MDA2. Moreover, consistent with our earlier observation that only low IgG titers are secreted by B-1 cells, no IgG Abs were found in lesions of these mice.

Monoclonal NA-17 also recognized MDA epitopes in atherosclerotic lesions of $\mathrm{Ldlr}^{-/-} \mathrm{Rag}^{-1-}$ mice (Figure 5C), whereas a control natural IgM did not. We also found that NA-17 could substantially inhibit the binding of MDA-LDL to J774 macrophages in a dose-dependent fashion, whereas a KLH-specific IgM showed only nonspecific inhibition (Figure 5D).

\section{Figure 7}

Natural IgM Abs against oxidation-specific epitopes facilitate apoptotic cell uptake by macrophages in vivo. (A) Percentage of macrophages that contained fluorescently labeled apoptotic thymocytes following i.p. injection. In RAG + PBS mice, about $27 \%$ of macrophages phagocytosed apoptotic cells. The percentage was significantly increased, to $33 \%$, in RAG + B-1 mice ( ${ }^{*} P<0.05$, unpaired $t$ test). Horizontal bars denote means. (B) Apoptotic thymocytes were preincubated with NA-17 or a control IgM that does not bind apoptotic cells before injection into Rag $1^{-/-}$mice. The phagocytic uptake was significantly different between the 3 groups ( $P=0.01,1$-way ANOVA). The percentage of macrophages taking up apoptotic cells was significantly increased when the apoptotic cells were preincubated with NA-17 (RAG + NA-17), compared with control IgM (RAG + control IgM) or without preincubation (RAG) $\left(32 \%\right.$ vs. $20 \%$ vs. $23 \%$; ${ }^{\star} P<0.05$, Bonferroni multiple comparison test). Horizontal bars denote means.

Natural IgM Abs in buman cord blood recognize oxidation-specific epitopes. We previously showed that IgG and IgM titers to oxidationspecific epitopes are present in nearly all adult human plasmas tested (4). However, we wanted to know whether the human IgM $\mathrm{NAb}$ repertoire displays binding to oxidation-specific epitopes similar to those observed in mice. IgM Abs found in umbilical cord blood are exclusively from the infant and are considered to represent the human equivalent of naive NAbs (31). Therefore, we characterized the binding properties of IgM in umbilical cord blood and in the respective maternal plasma samples. Importantly, umbilical cord plasma contained prominent IgM titers against MDA-LDL and OxLDL, but not native LDL or KLH (Figure 6A). In contrast, maternal plasma also contained IgM against $\mathrm{KLH}$, presumably due to exogenous antigen exposure (Figure 6A). Unlike IgM titers, IgG titers were found to be similar in maternal and umbilical cord plasma (data not shown), consistent with the ability of maternal IgG to be actively transferred across the placenta. As the total IgM titers were found to be generally higher in maternal samples, we calculated the ratios of oxidation-specific IgM to total IgM in individual samples. This revealed that MDA-LDL- and OxLDL-specific IgM - but not KLH-specific IgM - are relatively enriched in umbilical cord plasmas compared with maternal plasmas (Figure 6A), which typically also contain adaptive IgM Abs (e.g., against KLH).

We further showed that human umbilical cord IgM also bound to apoptotic cells (Figure 6B). This binding was at least in part mediated through the recognition of MDA epitopes, as, in the example shown, MDA-LDL competed for up to $45 \%$ of the binding of the umbilical cord IgM to apoptotic cells. Thus, oxidationspecific epitopes also appear to be a dominant target for natural IgM Abs in humans.

NAbs facilitate apoptotic cell uptake by macrophages in vivo. Natural IgM Abs and the MDA-LDL-specific NAb NA-17 recognize oxidation-specific epitopes on apoptotic cells (Figure 5A). When not promptly cleared, apoptotic cells are immunogenic and proinflammatory $(17,18)$. To test the hypothesis that these NAbs maintain homeostasis against oxidatively modified structures, we examined their ability to mediate enhanced clearance of apoptotic cells in vivo. Using a previously described model (32), we compared apoptotic cell uptake by macrophages in vivo in $\mathrm{Rag} 1^{-/-}$mice 10 weeks after reconstitution with B-1 cells or with PBS. Mice were injected i.p. with fluorescently labeled apoptotic thymocytes 4 days after induction of sterile peritonitis with thioglycollate. Macrophage 
uptake of apoptotic thymocytes was significantly enhanced in B-1 cell-reconstituted Rag1 $1^{-/}$mice compared with PBS controls (33\% vs. $27 \%, P<0.05$; Figure $7 \mathrm{~A}$ ).

To directly test the impact of an oxidation-specific $\mathrm{mAb}$, we preincubated the apoptotic cells with NA-17 or with a control IgM (specific for $\mathrm{KLH}$ ) before injection into $\mathrm{Rag} 1^{-/-}$mice. The percentage of macrophages that had taken up apoptotic thymocytes was similar in Rag $1^{-/-}$mice receiving apoptotic thymocytes preincubated with control IgM or untreated apoptotic thymocytes $(20 \%$ vs. $23 \%, P=$ NS; Figure $7 \mathrm{~B}$ ). In contrast, the phagocytic uptake increased to $32 \%$ when the apoptotic cells were preincubated with NA-17 $(P<0.05)$, demonstrating that binding of NA-17 facilitated apoptotic cell uptake by macrophages in vivo.

\section{Discussion}

Innate immune responses provide a vital and nonredundant role in the initial defense against invading pathogens and in maintaining homeostasis against a variety of self-antigens. The mediators of innate immunity are germline encoded, preformed, and utilize a limited set of PRRs to recognize a set of common PAMPs. It is now widely recognized that one class of PRRs, the so-called scavenger receptors, such as SRA-1 and -2, CD36, SR-B1, LOX-1, PSOX, and others, all recognize various oxidation-specific epitopes present on OxLDL and apoptotic cells (7). Indeed, recent evidence suggests that members of the innate TLR family also recognize such epitopes as well $(33,34)$. The soluble innate protein CRP also binds to the PC of OxPL present on OxLDL and apoptotic cells, as well as to $S$. pneumoniae (20). Thus, oxidation-specific epitopes - as a class - constitute an important PAMP recognized by innate immunity, consistent with the oxygen-centric nature of life itself.

NAbs are part of the humoral arc of innate immunity. They are the product of natural selection, exhibiting a genetically determined and stable repertoire that is largely independent of external antigenic stimuli (35-37). NAbs have been defined in various ways, for example, as the IgM present in normal plasma (38), but more stringently, as germline-encoded Abs that arise without any exogenous immune exposure or bacterial colonization of the gut (39). They are produced at tightly regulated and stable levels in healthy individuals and are found in all vertebrate species $(11,40)$. In normal, uninfected mice, most plasma IgM Abs are NAbs that are derived from innate B-1 cells, which differ from conventional $\mathrm{B}-2$ cells in surface phenotype; anatomic location; restricted use of $\mathrm{V}_{\mathrm{H}}$ genes that are minimally edited and reflective of germline usage; and, importantly, their capacity for self renewal (reviewed in refs. 11, 41, 42). Despite this, relatively little is known about B-1 cell ontogeny, development, and, surprisingly, function. Only recently has evidence been presented to support a lineage independent from that of B-2 cells (43-46). Although the mechanisms leading to selection and expansion of B-1 cells are unclear, it is now appreciated that antigen selection during the fetal and neonatal period leads to positive selection $(11,47,48)$. Since this appears to occur equally well in mice raised in germ-free environments $(35,49,50)$, this selection is of necessity made by endogenous "self-antigens." This is in contrast to developing, self-reactive B-2 cells, in which such antigen encounter early in life leads to negative selection via apoptosis (a process known as clonal deletion) or anergy. Thus, the repertoire of B-1 cells is selected to bind to evolutionarily important epitopes. Furthermore, because many, if not most, NAbs have dual specificities (11), typically with molecular epitopes on pathogens, an encounter later in life with such external pathogens could further serve to expand a given $\mathrm{B}-1$ cell clone $(12,51)$. The example given in the Introduction that E06/T15, which binds OxPL of OxLDL and apoptotic cells, also binds to the PCs present on the cell wall of many pathogens nicely illustrates this paradigm. We report now that as a class, oxidationspecific neoepitopes constitute a disproportionately large portion of such self-antigens.

In this article, we provide multiple lines of evidence suggesting that oxidation-specific epitopes are a dominant target of innate NAbs in both mice and humans. First, consistent with the view that the IgM pool of uninfected mice comprises NAbs (11), we show that SPF-maintained mice as well as germ-free mice maintained under strict gnotobiotic conditions contained prominent IgM titers to oxidation-specific epitopes compared with titers against recognized TI-2 antigens, such as $\alpha 1,3$-dextran. Consistent with this, there was a high frequency of ISCs to oxidation-specific epitopes, chiefly MDA, in spleens of wild-type mice. Of interest, the introduction of normal gut flora into the germ-free mice (conventionalized mice in Figure 1D) increased the titers to OxLDL, PC-BSA, 4-HNE-LDL, and $\alpha 1,3$-dextran. After birth, B-1 cells are typically thought to have minimal response to exogenous antigenic stimuli, but some, such as E06/T15 (which binds to the PC of OxPL in OxLDL and PC-BSA), are known to be responsive $(11,14)$.

Second, B-1 cells in culture, purified from naive uninfected mice, gave rise to an array of oxidation-specific IgM Abs, which were much more prevalent than IgM to the classic antigen $\alpha 1,3$-dex$\operatorname{tran}(42)$. Indeed, such IgM Abs were already detectable in supernatants of unstimulated B-1 cells in culture, and the production of these IgM Abs in response to stimulation, especially that of TLRs, increased to a greater extent than that of total IgM or IgM to $\alpha 1,3$-dextran. Moreover, by analogy to the robust anti-MDA responses in vivo, MDA-specific IgM Abs were the most dominant set of IgM Abs secreted in vitro, constituting up to $30 \%$ of total $\operatorname{IgM}$. A similar specificity was seen for the $\operatorname{IgM}$ found in plasma of Rag $1^{-/-}$mice following adoptive transfer of B-1 cells. Absorption studies demonstrated that $20 \%-35 \%$ of these IgM Abs had specificity for combinations of oxidation-specific epitopes, predominantly MDA-related epitopes. These data were further corroborated by ELISpot analysis of splenocytes of Rag $1^{-/-}$B-1 recipients, which demonstrates that ISCs against MDA-LDL accounted for $10 \%-12 \%$ of all ISCs in the spleens of the reconstituted mice, similar to the frequency found in spleens of wild-type mice. In addition, study of hybridomas generated from the spleens of the B-1 cell-reconstituted mice indicated that $20 \%-30 \%$ of all IgM-secreting clones bound to oxidation-specific epitopes, mostly MDA. Cloning and sequencing of a number of these confirmed their germline origin, as exemplified by the MDA-specific B-1 clone NA-17.

Third, these NAbs bound prominently to biologically relevant oxidation-specific epitopes. B-1 cell-derived IgM in the plasma of the reconstituted mice bound prominently to epitopes on apoptotic cells and to atherosclerotic lesions and colocalized with oxidationspecific epitopes in vivo in atherosclerotic lesions. Furthermore, monoclonal NA-17 bound prominently to apoptotic cells and to atherosclerotic lesions. These data strongly support the hypothesis that oxidation-specific epitopes are a dominant target of NAbs in mice, which in turn bind to biologically relevant self-antigens.

Fourth, IgM Abs in umbilical cord blood are solely of fetal origin and represent such naive NAbs in humans (31). The presence of an enriched titer of oxidation-specific IgM Abs in human umbilical cord blood, which bind to apoptotic cells in part via MDA-LDL 
epitopes, strongly suggests that oxidation-specific epitopes are an important target of NAbs in humans as well. Interestingly, Merbl et al., using a sensitive antigen-array screening technique, recently tested the specificities of autoreactive NAbs in human cord blood (31) and reported that the antigen to which such NAbs most commonly bound was native LDL. However, as noted in Results, though we often find low levels of binding to plated LDL, this cannot be competed by the same LDL in solution, suggesting that the LDL undergoes modification during the plating procedure (either structural, e.g., via oxidation, or even conformational). Indeed, the LDL used in the studies of Merbl et al. was bought from a commercial source, and we speculate that it may have been oxidized at the time of use. Thus, their data may be consistent with our observations that oxidation-specific epitopes are a dominant target of NAbs found in humans.

The apparent high prevalence of oxidation-specific epitopes as targets of NAbs in mice and humans likely reflects the ubiquitous presence of these epitopes consequent to oxidative events. Cells undergoing apoptosis and the microparticles shed from them are rich in oxidation-specific epitopes $(15,18,52)$. Because this process is universal, we speculate that they could be fundamental selecting antigens. Further, inflammatory events are associated with enhanced oxidative stress, and our different oxidation-specific Abs have been used to demonstrate the presence of these epitopes not only in atherosclerotic tissue, but in a variety of inflammatory settings, including renal, liver, and pulmonary disease (53-55), rheumatoid arthritis (our unpublished observation), CNS lesions found in multiple sclerosis (56), and Alzheimer disease (57), all of which would serve to provide stimulation later in life for particular B-1 cell clones. Finally, many if not most NAbs also react with microbes that contain the same or similar antigenic self-determinants, leading to cross-reactivity between self-determinants and microbial antigens. The classic natural IgM E06/T15, for example, binds PC of OxPL as well as PC as part of the capsular polysaccharide of pneumococci and many other microbes. Thus, postnatal stimulation of particular B-1 cell clones likely occurs upon exposure to molecular equivalents on pathogens.

Although the selection and expansion of B-1 cell clones clearly occurs in both fetal and postnatal life, the mechanisms leading to the activation of B-1 cells, conversion to plasma cells, and generation of NAbs are poorly understood. The mode of presentation of antigen that results in productive B- 1 cell expansion is generally considered to be independent of cognate $T$ cell help. Although some TI-2 multivalent antigens may lead to direct crosslinking of B cell receptor on B-1 cells and IgM production, in general most B-1 cell antigens do not (58). However, B-1 cells are known to be responsive to several non-cognate stimuli that also stimulate B-2 cells, including LPS and T cell cytokines such as IL-5. In our studies, we also found that NAbs specific for oxidation-specific epitopes are partially dependent on $\mathrm{T}$ cell help. First, we demonstrated in culture that IL-5 stimulated B- 1 cells to secrete IgM to oxidation-specific epitopes. Second, we found in vivo that anti-OxLDL IgM titers were lower in age-matched $\mathrm{T}$ cell-deficient mice compared with wild-type mice (Supplemental Figure 1) and that the B-1 cell transfer experiments with contaminating $\mathrm{T}$ cells resulted in more robust IgM titers in the recipients, including titers to OxLDL. Likely, the in vivo data can be explained in part by the secretion of IL-5, which we have previously shown to provide non-cognate help for the production of E06/T15 IgM in vivo in atherosclerotic mice (25). However, both T cell-deficient mice and Rag $1^{-/-}$recipients of pure B-1 cells exhibited IgM Abs against oxidation-specific epitopes, indicating that $T$ cell cytokines were not obligatory.

We also demonstrated that TLR4 and TLR2 agonists were particularly effective in stimulating the generation of IgM by B-1 cells in culture, an effect that was in large part MyD88 dependent. A similar observation was reported by Genestier et al. while this article was in preparation (59). Remarkably, however, our data indicate that TLR activation seems to have preferentially expanded the number of B-1 clones secreting IgM to oxidation-specific epitopes as compared with those secreting total IgM or IgM to $\alpha 1,3$ dextran (Figure 2A). This would imply that B-1 clones secreting such IgM are more responsive to activation by TLRs, suggesting evolutionary pressure linking generation of NAbs to oxidationspecific epitopes and activation of innate PRRs. This is intriguing, as we and others have provided data suggesting that aside from products of exogenous pathogens, there are endogenous antigens capable of stimulating such TLRs, including the oxidized moieties of OxLDL $(60,61)$. Obviously much study will be needed to explore such speculations.

NAbs have been said to be polyreactive and to bind to many autoantigens nonspecifically. However, we suggest that these observations may be confounded by the fact that a given NAb against a specific oxidation-specific epitope may bind to the same structural modification on many different proteins or even lipids. For example, E06 binds to the PCs moiety on the surface of bacteria, as well as to the PC headgroup of OxPL, which is found in OxLDL in both the lipid phase and covalently bound to apoB. E06 binds to OxPL covalently bound to apo(a) as well as OxPL in the lipid phase of $\mathrm{Lp}(\mathrm{a})$ (62). It also binds to the OxPLs present on the cell surface of apoptotic cells (19) and to the OxPLs generated in the lungs of mice and humans infected with viruses (55). Thus, it binds not only to atherosclerotic lesions, but to a large number of tissues in which inflammation exists, presumably to the PC epitope generated as result of lipid peroxidation and/or apoptosis. If one did not know the true identity of the epitope of the NAb E06, one would say this Ab was "polyreactive." In this article, we identify an even more prominent set of natural IgM directed against MDA epitopes (which are in fact a complex set of related epitopes) that are generated as a consequence of lipid peroxidation. The highly reactive MDA may similarly modify a wide range of substrates, including proteins, lipids, and even DNA (63), generating MDA neoepitopes in a variety of pathophysiological events, such as ischemia and reperfusion, diabetes, and atherosclerosis, as well as inflammatory events in the brain (64). Indeed, we speculate that many of the socalled self-antigens that have been reported to be targets of NAbs may well be modified self-antigens.

Functionally, NAbs play an important role in providing the first line of defense against viral and bacterial pathogens (65-67). NAbs also have important activities in homeostasis (68), including immunoregulatory functions, tumor surveillance, and recognition and removal of senescent cells, cell debris, and other self-antigens, which if persisting would be proinflammatory and immunogenic. Indeed, mice that cannot secrete IgM Abs and thus lack natural IgM have been found to more readily develop autoimmune disease when crossed on a susceptible background (69). Here we demonstrate that both murine natural IgM as well as human umbilical cord IgM bind apoptotic cells, but not normal cells, in part through binding to oxidation-specific epitopes on their cell surface. IgM Abs in general have been shown to be 
required in complement-mediated clearance of apoptotic cells (70). In the present work we explicitly demonstrate that pure IgM NAbs facilitate macrophage uptake of apoptotic cells in vivo and, specifically, that the MDA-specific NAb NA-17 similarly promotes clearance. Previously, the OxPL-specific NAb E06/T15 was also shown to facilitate efficient complement-mediated phagocytosis of apoptotic cells in vivo (71). These data demonstrate an important role for oxidation-specific natural IgM in mediating clearance of apoptotic cells. There are likely many other roles as well. Oxidized lipids have increasingly been shown to be proinflammatory. Apoptotic cells and their apoptotic blebs can activate endothelial cells, and E06 can block these effects $(19,52)$. Recently it was shown that multiple lung pathogens, such as chemical agents, $\mathrm{H} 5 \mathrm{~N} 1$ avian flu, or SARS, which are associated with high lethality due to acute respiratory distress syndrome, induce robust OxPL formation in the lung. In turn, the OxPLs induce lung injury and cytokine production by lung macrophages, and this latter effect was ameliorated by E06/T15 in vitro (55). As another example, we have previously shown that enhancing the titer of E06/T15 is atheroprotective (14), in part through inhibiting the uptake of OxLDL by macrophages. We now show that NA-17 also has the ability to inhibit binding of MDA-LDL in a similar manner. A great deal of work will be needed to assess the overall functional role of such oxidation-specific NAbs in atherosclerosis in particular and in homeostasis in general.

In conclusion, oxidation-specific epitopes constitute a previously unrecognized but important target of NAbs, and of innate immunity in general. NAbs may be beneficial not only for defense against pathogens but also in identifying altered-self produced as a result of oxidative stress under inflammatory events. Understanding their role in health and disease may lead to novel diagnostic and possibly therapeutic approaches to deal with consequences of oxidative stress such as atherogenesis.

\section{Methods}

Animals. C57BL/6 and Rag1 $1^{-/}$mice (The Jackson Laboratory) and Ldlr/Rag $1^{-/-}$mice (a gift from Godfrey Getz and Katherine Reardon, University of Chicago, Chicago, Illinois, USA), all on C57BL/6 background, were bred and maintained in our colony under SPF conditions unless otherwise noted. Plasma was obtained from 6-week-old female C57BL/6 T cell receptor $\alpha^{-/-}\left(\mathrm{Tcra}^{-/-}\right)$mice (courtesy of Stephen Hedrick, UCSD).

Fourteen- to 16-week-old germ-free Swiss Webster mice were maintained at the Göteborg University vivarium under strict gnotobiotic conditions. Sterility was monitored by stool culturing and PCR for bacterial DNA. Conventionally raised mice were transferred to gnotobiotic isolators at weaning and fed the same autoclaved chow diet. To obtain conventionalized mice, 13- to 14-week-old germ-free mice were colonized with cecal content from 12-week-old conventionally raised Swiss Webster donors for 14 days.

All experimental protocols were approved by the Animal Subjects Committee at UCSD; care and use of the germ-free mice was approved by the Göteborg University Animal Studies Committee.

B-1 cell isolation. Peritoneal exudate cells (PECs) from 6 -to 15-week-old naive $\mathrm{C} 57 \mathrm{BL} / 6$ mice were harvested by peritoneal lavage using ice-cold PBS supplemented with $1 \%$ heat-inactivated FCS (Invitrogen). PECs were incubated with an anti-Fcy receptor $\mathrm{mAb}$ (clone 2.4G2; BD Biosciences - Pharmingen) for 15 minutes at $4^{\circ} \mathrm{C}$ before being stained with fluorescently labeled $\mathrm{mAbs}$ to block nonspecific binding. PECs were stained with R-PE-labeled anti-CD19 (1D3), FITC-labeled anti-CD23 (B3B4), and in some experiments PE-Cy5-labeled anti-CD3 (clone 145-2C11) (all from BD Biosciences - Pharmingen). B-1 cells were sorted to greater than
$99 \%$ purity using a FACSVantage SE cell sorter $(\mathrm{BD})$ as the $\mathrm{CD}^{-}, \mathrm{CD}^{-} 9^{+}$, and $\mathrm{CD}_{23}^{-}$population.

$B-1$ cell cultures. Purified B- 1 cells were seeded at $1 \times 10^{6}$ cells per well in 24-well flat-bottom plates in culture medium (RPMI 1640 medium containing 10\% heat-inactivated FCS, $10 \mathrm{mM}$ HEPES buffer, $2 \mathrm{mM}$ L-glutamine, $0.05 \mathrm{mM}$ 2-mercaptoethanol, $50 \mu \mathrm{g} / \mathrm{ml}$ gentamicin) in the presence and absence of the specific TLR4 agonist $\mathrm{KdO}_{2}$-Lipid A (100 ng/ml; Avanti Polar Lipids), a combination of the TLR2 agonists Pam3CSK4 $(300 \mathrm{ng} / \mathrm{ml})$ and FSL-1 $(1 \mu \mathrm{g} / \mathrm{ml})$ (Invivogen), or vehicle control in triplicate in a final volume of $500 \mu \mathrm{l}$. Cells were incubated at $37^{\circ} \mathrm{C} / 5 \% \mathrm{CO}_{2}$ for up to 7 days.

Adoptive transfer of B-1 cells. B-1 cells were isolated from the peritoneum of 6- to 15 -week-old female donor C57BL/6 mice as described above. Purified B- 1 cells were resuspended in PBS, and 0.5 or $1 \times 10^{6}$ cells in $200 \mu$ l were injected into the peritoneal cavity of 6 - to 15 -week-old Rag $1^{-/-}$mice. Three to 4 donor mice were used for each recipient. Control Rag $1^{-/-}$mice received an equal volume of PBS. Blood was collected via the retro-orbital plexus from recipient mice before and 4 and 10 weeks after transfer.

Flow cytometry. At the time of sacrifice, PECs and splenocytes were resuspended in staining buffer. After blocking with a specific anti-Fc $\gamma$ receptor $\mathrm{mAb}(2.4 \mathrm{G} 2)$ for 15 minutes at $4^{\circ} \mathrm{C}, 10^{6}$ cells were stained with fluorescently labeled mAbs specific for various surface markers (FITC-labeled anti-CD11b/Mac-1 [M1/70], PE-labeled anti-CD5, PerCP-Cy5.5-labeled anti-CD19 [1D3]; PE-labeled anti-mouse CD43 [S7], and APC- or FITClabeled anti-mouse IgM [II/41]; all from BD Biosciences - Pharmingen) in $100-\mu \mathrm{l}$ volumes of staining buffer for 30 minutes at $4^{\circ} \mathrm{C}$ in darkness, followed by extensive washing. Cell populations were analyzed on a BD FACSCalibur or FACScan instrument. More than $0.5 \times 10^{5}$ cells were analyzed per sample, with dead cells excluded by forward and side scatter. Surface marker analysis was performed using FlowJo software (Tree Star Inc.).

Measurement of Ab titers. Specific Ab titers to given antigens in plasma or cell culture supernatants were determined by chemiluminescent ELISA as previously described $(14,23)$. Ab dilution curves of $\mathrm{Ab}$ binding to plated antigens were determined by serial dilutions of plasma or culture supernatant, and a titer was defined as the highest dilution that yielded binding that was 2-fold greater than the background level. Purified rat anti-mouse IgM (II/41; BD Biosciences - Pharmingen) was used as capture Ab to measure total IgM levels. AP-labeled goat anti-mouse IgM ( $\mu$ chain specific) and anti-mouse IgG ( $\gamma$ chain specific) (Sigma-Aldrich) were used as detection $\mathrm{Abs}$, as well as biotinylated rat anti-mouse IgM (R6-60.2; BD Biosciences - Pharmingen). To detect other Ig isotypes, rat anti-mouse IgG1 (A85-3), IgG2a/c (R11-89), IgG2b (R9-91), IgG3 (R2-38), and IgA (C10-3) were used as capture Abs; biotin-conjugated rat anti-mouse IgG1 (A85-1), IgG2a/c (R19-15), IgG2b (R12-3), IgG3 (R40-82), and IgA (C10-1) (all from BD Biosciences - Pharmingen) were used as secondary Abs. To detect the levels of E06, a T15-specific anti-idiotype Ab (AB1-2) (72) was used as capture Ab, followed by incubation with AP-labeled goat anti-mouse IgM. Biotin-conjugated Abs were then detected with AP-conjugated neutravidin (Pierce, Thermo Scientific). Mouse anti-human IgG (G18-145) and IgM (G20-127; $\mathrm{BD}$ Biosciences - Pharmingen) were used as capture Abs to measure total IgM and IgG levels in humans. AP-labeled goat anti-human IgG and IgM (A3187 and A3437; Sigma-Aldrich) were used as detection Abs. The following antigens were prepared as described previously (29): copper sulfate-oxidized LDL (CuOx-LDL), 4-HNE-LDL, MDA-LDL prepared from human LDL, and 4-HNE-MSA prepared from MSA. MAA-BSA was prepared as described previously (73). $\alpha 1,3$-dextran was a gift from John F. Kearney (University of Alabama at Birmingham, Birmingham, Alabama, USA). PCBSA and PC-KLH were from Biosearch Technologies Inc., and KLH was from Pierce Biotechnology.

Immunocompetition assays. The specificity of IgM Abs binding to MDALDL was determined by competition immunoassays as described previ- 
ously $(14,23)$. Plasma from B-1 cell-recipient Rag $1^{-/-}$mice was pooled and diluted to 1:100 for MDA-LDL and 1:200 for CuOx-LDL, or B-1 cell supernatants were incubated overnight at $4{ }^{\circ} \mathrm{C}$ in the presence and absence of increasing concentrations of competitors. Samples were then centrifuged at $15,800 \mathrm{~g}$ for 45 minutes at $4^{\circ} \mathrm{C}$ and supernatants analyzed for binding to the respective antigen by chemiluminescent ELISA.

To determine the percentage of total IgM in plasma binding to specific antigens, plasma samples were diluted in 1\% BSA-TBS to yield a limiting dilution for each antigen, as determined in preliminary experiments. Diluted plasma samples were incubated overnight at $4^{\circ} \mathrm{C}$ in the absence or presence of individual or combinations of antigens at a final concentration of $250 \mu \mathrm{g} / \mathrm{ml}$. Thereafter, samples were centrifuged at $15,800 \mathrm{~g}$ for 45 minutes at $4{ }^{\circ} \mathrm{C}$ to pellet immune complexes and supernatants analyzed for total IgM content by chemiluminescent ELISA, using a monoclonal rat anti-mouse IgM to capture and a polyclonal alkaline phosphatase-labeled (AP-labeled) goat anti-mouse IgM ( $\mu$ chain specific) for detection. Data were calculated as the amount of IgM remaining in the supernatant after absorption, expressed as a percentage of total IgM. In some experiments, we calculated the apparent Ab avidity of IgM binding to a given antigen by analysis of the competition assays using the Klotz method (23).

ELISpot assay. The frequencies of total and antigen-specific IgM-secreting splenocytes were quantified by ELISpot assay as described previously (14). Splenocytes were suspended in culture medium at $2 \times 10^{5}$ and $1 \times 10^{5}$ cells/ $100 \mu \mathrm{l}$ and cultured in triplicate in washed and blocked 96-well MultiScreen-HA sterile nitrocellulose plates (Millipore) that had been coated overnight with $4 \mu \mathrm{g} / \mathrm{ml}$ of rat anti-mouse IgM (II/41), MDA-LDL, OxLDL, 4-HNE-LDL, or $\alpha 1,3$-dextran. After 22 hours incubation at $37^{\circ} \mathrm{C} / 5 \% \mathrm{CO}_{2}$, cells were removed by washing, and ISCs were detected using biotinylated rat anti-mouse IgM, followed by HRP-streptavidin (Zymed Laboratories Inc., Invitrogen). Plates were developed using a tetramethylbenzidine membrane substrate system (KPL), and spots were quantified using an automated ImmunoSpot Image Analyzer (Cellular Technology Ltd.).

Apoptotic cells and immunofluorescence microscopy. Thymocytes harvested from C57BL/6 mice were cultured in cell culture medium and induced to undergo apoptosis by $10 \mathrm{ng} / \mathrm{ml}$ PMA (Sigma-Aldrich) for 16 hours as described previously (18). Plasma, NA-17, or control IgM (C48-6, antiKLH; BD Biosciences - Pharmingen) diluted in 1\% BSA-PBS was incubated with apoptotic thymocytes for 30 minutes at $4{ }^{\circ} \mathrm{C}$. Cells were washed and incubated with FITC-labeled rat anti-mouse IgM (II/41) in 1\% BSAPBS for 30 minutes and then washed again. For FACS analysis, cells were incubated with annexin $\mathrm{V}$ and 7-AAD (BD Biosciences - Pharmingen) for 15 minutes and immediately analyzed by BD FACSCanto. Umbilical plasma IgM binding to apoptotic Jurkat cells was detected by FITC-conjugated goat anti-human IgM (Jackson ImmunoResearch Laboratories Inc.). Apoptosis of Jurkat cells was induced with UV irradiation at $20 \mathrm{~mJ} / \mathrm{cm}^{2}$, followed by further incubation of the cells in medium for 14-16 hours before use. For immunofluorescence microscopy studies, cells were incubated with $2 \mu \mathrm{g} / \mathrm{ml}$ Hoechst dye (Sigma-Aldrich) for 10 minutes, fixed with $2 \%$ paraformaldehyde, and spun down on glass slides using a cytospin (StatSpin). Images were captured by deconvolution microscopy using a DeltaVision deconvolution microscopic system operated by softWoRx software (Applied Precision) as described previously (18).

Immunocytochemistry. Frozen-embedded sections (embedded in TissueTek OCT compound [Sakura Finetek]) of aortic origin from cholesterol-fed Ldlr ${ }^{-/}$Rag $1^{-/-}$mice were fixed with methanol, blocked with $2 \%$ goat serum, and stained with $5 \mu \mathrm{g} / \mathrm{ml} \mathrm{MDA} 2,1.6 \mu \mathrm{g} / \mathrm{ml} \mathrm{EN2}$, or $0.8 \mu \mathrm{g} / \mathrm{ml} \mathrm{NA}-17$ (29), followed by a biotinylated goat anti-mouse IgG or anti-mouse IgM (Jackson ImmunoResearch Laboratories Inc.) to detect endogenous NAbs in the lesions. A Vectastain ABC-AP kit and a Vector Red AP chromogenic substrate (Vector Laboratories) were used to visualize the Ab staining. Slides were counterstained with Weigert's iron hematoxylin (Richard-Allan Scientific, Thermo Scientific). Immunostaining of adjacent sections in the absence of primary $\mathrm{Ab}$ was used as a negative control.

Cloning and genetic analysis of hybridoma NA-17. Spleens of Rag $1^{-/-}$mice reconstituted with B-1 cells were used to prepare B-1-derived hybridomas using techniques established in our laboratory (29). Productive hybridomas, by default, should only secrete NAbs. In brief, splenocytes from 4 Rag1-/- B-1 recipients were fused with the P3 $\times 63 \mathrm{Ag} 8.653 .1$ myeloma cell line. Primary screening of supernatants was performed after 10 days of growth for the ability to secrete IgM and subsequently for IgM binding to MDA-LDL by chemiluminescent ELISA. Selected hybridomas were then cloned by limiting dilution (8). Here we report on clone NA-17, which was confirmed by DNA sequencing to be a NAb. Cloning and sequence analysis of $\operatorname{IgM} N A-17$ hybridoma $V_{H}$ and $V_{L}$ was accomplished as previously described (12).

Human samples. Blood samples from healthy pregnant women and their newborn infants delivering at Magee-Womens Hospital (Pittsburgh, Pennsylvania, USA) were collected as part of a prospective study of preeclampsia, approved by the Institutional Review Board of the University of Pittsburgh, for which written informed consent was received from the mothers. Maternal samples were collected in EDTA before delivery (mean, 10.5 hours) and cord venous samples by sterile aspiration of the umbilical vein after delivery of the infant. Samples were processed and aliquoted within 3 hours of collection and stored at $-70^{\circ} \mathrm{C}$ until use. Ten uncomplicated nulliparous maternal-infant pairs were selected for this preliminary analysis. The mothers (50\% of mixed European descent and 50\% African American) were $23.4 \pm 5.4$ years of age, $39.9 \pm 1.2$ weeks gestational age (values are mean and SD). There were 6 male and 4 female infants.

In vivo uptake of apoptotic cells by peritoneal macrophages. The in vivo clearance of apoptotic cells was assessed using a modification of the method described by Taylor et al. (32). Rag $1^{-/-}$mice, adoptively transferred with B-1 cells or with PBS, were injected i.p. with $1 \mathrm{ml}$ of sterile $3 \%$ thioglycollate to induce sterile peritonitis $15-19$ weeks after B-1 cell transfer. Four days later, the mice were injected i.p. with $20 \times 10^{6}$ fluorescently labeled apoptotic thymocytes (using 5-CMFDA; Molecular Probes, Invitrogen) in $200 \mu \mathrm{l} \mathrm{PBS}$. The mice were sacrificed 40 minutes after injection, and peritoneal cells were recovered by lavage with $10 \mathrm{ml}$ of ice-cold PBS with $1 \%$ heat-inactivated FBS/10 mM EDTA. Macrophages were labeled with PE-conjugated F4/80 (BM8; eBioscience) and macrophage-specific uptake of apoptotic cells analyzed by FACS. Phagocytosis was expressed as the percentage of macrophages ingesting apoptotic cells. To test the ability of NA-17 to mediate enhanced uptake, fluorescently labeled apoptotic thymocytes were preincubated with cultured NA-17 hybridoma supernatant or anti-KLH IgM (C48-6; BD Biosciences - Pharmingen) at $5 \mu \mathrm{g} / \mathrm{ml}$ for 1 hour, washed, and then injected into 8- to 10-week-old Rag 1-/- mice.

Macrophage binding assay. Binding of biotinylated MDA-LDL to J774 macrophages plated in microtiter wells was assessed by a chemiluminescent binding assay as described previously (60). Biotinylated MDA-LDL $(2 \mu \mathrm{g} / \mathrm{ml})$ was incubated in the absence or presence of NA-17 or anti-KLH IgM (C48-6; BD Biosciences - Pharmingen) at different concentrations overnight. Samples were then centrifuged at $15,800 \mathrm{~g}$ for 45 minutes at $4{ }^{\circ} \mathrm{C}$. The harvested supernatants were then added to macrophages and the binding of biotinylated MDA-LDL determined by ELISA.

Statistics. Statistical tests used to analyze for significance are described in the figure legends. The tests used were: 1-way or repeated measures ANOVA with Tukey-Kramer multiple comparison tests; Wilcoxon matched-pairs test; and paired $t$ test, using 2-tailed levels of significance. Results were analyzed with InStat 3 for Macintosh (GraphPad Software). A P value less than 0.05 was considered significant. 


\section{Acknowledgments}

This work was supported by American Heart Association (AHA) Scientist Development Awards 0530159N (to Y.I. Miller), 0630228N (to K. Hartvigsen), and 0430127N (to P.X. Shaw) and AHA Postdoctoral Award 0625133 Y (to L.F. Hansen); NIH grants HL086559 (to J.L. Witztum), P50 HL056989 (to J.L. Witztum), and P01 HL088093 (to J.L. Witztum, Y.I. Miller, and C.J. Binder); the Austrian Academy of Sciences (to C.J. Binder); the Fondation Leducq (to J.L. Witztum and C.J. Binder); and grants from the Swedish Heart and Lung Foundation, the Swedish Society of Medicine, and the Gothenburg Medical Society (to L. Fogelstrand).
Received for publication July 16, 2008, and accepted in revised form February 25, 2009.

Address correspondence to: Joseph L. Witztum, University of California, San Diego, Basic Science Building, Room 1080, 9500 Gilman Drive, La Jolla, California 92093, USA. Phone: (858) 534-4347; Fax: (858) 534-2005; E-mail: jwitztum@ucsd.edu. Or to: Christoph J. Binder, CeMM and Department of Medical and Chemical Laboratory Diagnostics, Medical University of Vienna, Währinger Gürtel 18-20, A-1090 Vienna, Austria. Phone: 43-1-40400-6441; Fax: 43-1-40400-2097; E-mail: christoph.binder@meduniwien.ac.at.
1. Binder, C.J., et al. 2002. Innate and acquired immunity in atherogenesis. Nat. Med. 8:1218-1226.

2. Hansson, G.K., and Libby, P. 2006. The immune response in atherosclerosis: a double-edged sword. Nat. Rev. Immunol. 6:508-519.

3. Getz, G.S., Vanderlaan, P.A., and Reardon, C.A. 2007. The immune system and murine atherosclerosis. Curr. Drug Targets. 8:1297-1306.

4. Binder, C.J., et al. 2005. The role of natural antibodies in atherogenesis. J. Lipid Res. 46:1353-1363.

5. Boullier, A., et al. 2005. Phosphocholine as a pattern recognition ligand for CD36. J. Lipid Res. 46:969-976

6. Gillotte-Taylor, K., Boullier, A., Witztum, J.L., Steinberg, D., and Quehenberger, O. 2001. Scavenger receptor class B type I as a receptor for oxidized low density lipoprotein. J. Lipid Res. 42:1474-1482.

7. Plüddemann, A., Neyen, C., and Gordon, S. 2007. Macrophage scavenger receptors and host-derived ligands. Methods. 43:207-217.

8. Palinski, W., et al. 1996. Cloning of monoclonal autoantibodies to epitopes of oxidized lipoproteins from apolipoprotein E-deficient mice. Demonstration of epitopes of oxidized low density lipoprotein in human plasma. J. Clin. Invest. 98:800-814.

9. Hörkkö, S., Miller, E., Branch, D.W., Palinski, W., and Witztum, J.L. 1997. The epitopes for some antiphospholipid antibodies are adducts of oxidized phospholipid and beta2 glycoprotein 1 (and other proteins). Proc. Natl. Acad. Sci. U. S. A. 94:10356-10361

10. Boullier, A., et al. 2000. The binding of oxidized low density lipoprotein to mouse CD36 is mediated in part by oxidized phospholipids that are associated with both the lipid and protein moieties of the lipoprotein. J. Biol. Chem. 275:9163-9169.

11. Baumgarth, N., Tung, J.W., and Herzenberg, L.A. 2005. Inherent specificities in natural antibodies: a key to immune defense against pathogen invasion. Springer Semin. Immunopathol. 26:347-362.

12. Shaw, P.X., et al. 2000. Natural antibodies with the T15 idiotype may act in atherosclerosis, apoptotic clearance, and protective immunity. J. Clin. Invest. 105:1731-1740.

13. Briles, D.E., Forman, C., Hudak, S., and Claflin, J.L. 1982. Anti-phosphorylcholine antibodies of the T15 idiotype are optimally protective against Streptococcus pneumoniae. J. Exp. Med. 156:1177-1185.

14. Binder, C.J., et al. 2003. Pneumococcal vaccination decreases atherosclerotic lesion formation: molecular mimicry between Streptococcus pneumoniae and oxidized LDL. Nat. Med. 9:736-743.

15. Kagan, V.E., et al. 2004. Oxidative lipidomics of apoptosis: redox catalytic interactions of cytochrome $\mathrm{c}$ with cardiolipin and phosphatidylserine. Free Radic. Biol. Med. 37:1963-1985.

16. Greenberg, M.E., et al. 2006. Oxidized phosphatidylserine-CD36 interactions play an essential role in macrophage-dependent phagocytosis of apoptotic cells. J. Exp. Med. 203:2613-2625.

17. Savill, J., Dransfield, I., Gregory, C., and Haslett, C. 2002. A blast from the past: clearance of apoptotic cells regulates immune responses. Nat. Rev. Immu- nol. 2:965-975.

18. Chang, M.K., et al. 2004. Apoptotic cells with oxidation-specific epitopes are immunogenic and proinflammatory. J. Exp. Med. 200:1359-1370.

19. Chang, M.K., et al. 1999. Monoclonal antibodies against oxidized low-density lipoprotein bind to apoptotic cells and inhibit their phagocytosis by elicited macrophages: evidence that oxidation-specific epitopes mediate macrophage recognition. Proc. Natl. Acad. Sci. U. S. A. 96:6353-6358.

20. Chang, M.K., Binder, C.J., Torzewski, M., and Witztum, J.L. 2002. C-reactive protein binds to both oxidized LDL and apoptotic cells through recognition of a common ligand: phosphorylcholine of oxidized phospholipids. Proc. Natl. Acad. Sci.U.S. A. 99:13043-13048.

21. Kawahara, T., Ohdan, H., Zhao, G., Yang, Y.G., and Sykes, M. 2003. Peritoneal cavity B cells are precursors of splenic IgM natural antibody-producing cells. J. Immunol. 171:5406-5414.

22. Hörkkö, S., et al. 1999. Monoclonal autoantibodies specific for oxidized phospholipids or oxidized phospholipid-protein adducts inhibit macrophage uptake of oxidized low-density lipoproteins. J. Clin. Invest. 103:117-128.

23. Friguet, B., et al. 1985. Measurements of the true affinity constant in solution of antigen-antibody complexes by enzyme-linked immunosorbent assay. J. Immunol. Methods. 77:305-319.

24. Masmoudi, H., Mota-Santos, T., Huetz, F., Coutinho, A., and Cazenave, P.A. 1990. All T15 Id-positive antibodies (but not the majority of VHT15+ antibodies) are produced by peritoneal CD5+ B lymphocytes. Int. Immunol. 2:515-520.

25. Binder, C.J., et al. 2004. IL-5 links adaptive and natural immunity specific for epitopes of oxidized LDL and protects from atherosclerosis. J. Clin. Invest. 114:427-437.

26. Manz, R.A., Hauser, A.E., Hiepe, F., and Radbruch, A. 2005. Maintenance of serum antibody levels. Annu. Rev. Immunol. 23:367-386.

27. Shaw, P.X., et al. 2001. Human-derived anti-oxidized LDL autoantibody blocks uptake of oxidized LDL by macrophages and localizes to atherosclerotic lesions in vivo. Arterioscler. Thromb. Vasc. Biol. 21:1333-1339.

28. Tabas, I. 2005. Consequences and therapeutic implications of macrophage apoptosis in atherosclerosis: the importance of lesion stage and phagocytic efficiency. Arterioscler. Thromb. Vasc. Biol. 25:2255-2264.

29. Ylä-Herttuala, S., et al. 1989. Evidence for the presence of oxidatively modified low density lipoprotein in atherosclerotic lesions of rabbit and man. J. Clin. Invest. 84:1086-1095.

30. Palinski, W., et al. 1990. Antisera and monoclonal antibodies specific for epitopes generated during oxidative modification of low density lipoprotein. Arteriosclerosis. 10:325-335.

31. Merbl, Y., Zucker-Toledano, M., Quintana, F.J., and Cohen, I.R. 2007. Newborn humans manifest autoantibodies to defined self molecules detected by antigen microarray informatics. J. Clin. Invest.
117:712-718

32. Taylor, P.R., et al. 2000. A hierarchical role for classical pathway complement proteins in the clearance of apoptotic cells in vivo. J. Exp. Med. 192:359-366.

33. Miller, Y.I., et al. 2005. Toll-like receptor 4-dependent and -independent cytokine secretion induced by minimally oxidized low-density lipoprotein in macrophages. Arterioscler. Thromb. Vasc. Biol. 25:1213-1219.

34. Miller, Y.I., Chang, M.K., Binder, C.J., Shaw, P.X., and Witztum, J.L. 2003. Oxidized low density lipoprotein and innate immune receptors. Curr. Opin. Lipidol. 14:437-445.

35. Haury, M., et al. 1997. The repertoire of serum IgM in normal mice is largely independent of external antigenic contact. Eur. J. Immunol. 27:1557-1563.

36. Vasconcellos, R., Nobrega, A., Haury, M., Viale, A.C., and Coutinho, A. 1998. Genetic control of natural antibody repertoires: I. IgH, MHC and TCR beta loci. Eur. J. Immunol. 28:1104-1115.

37. Mouthon, L., et al. 1995. Invariance and restriction toward a limited set of self-antigens characterize neonatal IgM antibody repertoires and prevail in autoreactive repertoires of healthy adults. Proc. Natl. Acad. Sci. U. S. A. 92:3839-3843.

38. Jayasekera, J.P., Moseman, E.A., and Carroll, M.C. 2007. Natural antibody and complement mediate neutralization of influenza virus in the absence of prior immunity. J. Virol. 81:3487-3494.

39. Hooijkaas, H., Benner, R., Pleasants, J.R., and Wostmann, B.S. 1984. Isotypes and specificities of immunoglobulins produced by germ-free mice fed chemically defined ultrafiltered "antigen-free" diet. Eur. J. Immunol. 14:1127-1130.

40. Avrameas, S. 1991. Natural autoantibodies: from "horror autotoxicus" to "gnothi seauton." Immunol. Today. 12:154-159.

41. Hardy, R.R., and Hayakawa, K. 2005. Development of $\mathrm{B}$ cells producing natural autoantibodies to thymocytes and senescent erythrocytes. Springer Semin. Immunopathol. 26:363-375.

42. Kearney, J.F. 2005. Innate-like B cells. Springer Semin. Immunopathol. 26:377-383.

43. Montecino-Rodriguez, E., Leathers, H., and Dorshkind, K. 2006. Identification of a B-1 B cell-specified progenitor. Nat. Immunol. 7:293-301.

44. Herzenberg, L.A., and Tung, J.W. 2006. B cell lineages: documented at last! Nat. Immunol. 7:225-226.

45. Tung, J.W., Mrazek, M.D., Yang, Y., Herzenberg, L.A., and Herzenberg, L.A. 2006. Phenotypically distinct $\mathrm{B}$ cell development pathways map to the three B cell lineages in the mouse. Proc. Natl. Acad. Sci. U. S. A. 103:6293-6298.

46. Dorshkind, K., and Montecino-Rodriguez, E. 2007. Fetal B-cell lymphopoiesis and the emergence of B-1-cell potential. Nat. Rev. Immunol. 7:213-219.

47. Hardy, R.R., Wei, C.J., and Hayakawa, K. 2004. Selection during development of VH11+ B cells: a model for natural autoantibody-producing CD5+ B cells. Immunol. Rev. 197:60-74.

48. Qian, Y., et al. 2006. Autoreactive MZ and B-1 B-cell activation by Faslpr is coincident with an increased frequency of apoptotic lymphocytes and a defect in 
macrophage clearance. Blood. 108:974-982.

49. Bos, N.A., et al. 1987. Early development of Igsecreting cells in young of germ-free BALB/c mice fed a chemically defined ultrafiltered diet. Cell. Immunol. 105:235-245.

50. Thurnheer, M.C., Zuercher, A.W., Cebra, J.J., and Bos, N.A. 2003. B1 cells contribute to serum IgM, but not to intestinal $\operatorname{IgA}$, production in gnotobiotic Ig allotype chimeric mice. J. Immunol. 170:4564-4571.

51. Kearney, J.F. 2000. Immune recognition of OxLDL in atherosclerosis. J. Clin. Invest. 105:1683-1685.

52. Huber, J., et al. 2002. Oxidized membrane vesicles and blebs from apoptotic cells contain biologically active oxidized phospholipids that induce monocyte-endothelial interactions. Arterioscler. Thromb. Vasc. Biol. 22:101-107.

53. Houglum, K., et al. 1997. Excess iron induces hepatic oxidative stress and transforming growth factor beta1 in genetic hemochromatosis. Hepatology. 26:605-610.

54. Horie, K., et al. 1997. Immunohistochemical colocalization of glycoxidation products and lipid peroxidation products in diabetic renal glomerular lesions. Implication for glycoxidative stress in the pathogenesis of diabetic nephropathy. J. Clin. Invest. 100:2995-3004.

55. Imai, Y., et al. 2008. Identification of oxidative stress and Toll-like receptor 4 signaling as a key pathway of acute lung injury. Cell. 133:235-249.

56. Newcombe, J., Li, H., and Cuzner, M.L. 1994. Low density lipoprotein uptake by macrophages in multiple sclerosis plaques: implications for pathogenesis. Neuropathol. Appl. Neurobiol. 20:152-162.
57. Dei, R, et al. 2002. Lipid peroxidation and advanced glycation end products in the brain in normal aging and in Alzheimer's disease. Acta Neuropathol. (Berl.). 104:113-122.

58. Berland, R., and Wortis, H.H. 2002. Origins and functions of B-1 cells with notes on the role of CD5. Annu. Rev. Immunol. 20:253-300.

59. Genestier, L., et al. 2007. TLR agonists selectively promote terminal plasma cell differentiation of B cell subsets specialized in thymus-independent responses. J. Immunol. 178:7779-7786.

60. Miller, Y.I., et al. 2003. Minimally modified LDL binds to CD14, induces macrophage spreading via TLR4/MD-2, and inhibits phagocytosis of apoptotic cells. J. Biol. Chem. 278:1561-1568.

61. Bae, Y.S., et al. 2009. Macrophages generate reactive oxygen species in response to minimally oxidized low-density lipoprotein. Toll-like receptor 4- and spleen tyrosine kinase-dependent activation of NADPH oxidase 2. Circ. Res. 104:210-218.

62. Bergmark, C., et al. 2008. A novel function of lipoprotein [a] as a preferential carrier of oxidized phospholipids in human plasma. J. Lipid Res. 49:2230-2239.

63. Blair, I.A. 2008. DNA-adducts with lipid peroxidation products. J. Biol. Chem. 283:15545-15549.

64. Esterbauer, H., Schaur, R.J., and Zollner, H. 1991. Chemistry and biochemistry of 4-hydroxynonenal, malonaldehyde and related aldehydes. Free Radic. Biol. Med. 11:81-128.

65. Ochsenbein, A.F., et al. 1999. Control of early viral and bacterial distribution and disease by natural antibodies. Science. 286:2156-2159.

66. Boes, M., Prodeus, A.P., Schmidt, T., Carroll,
M.C., and Chen, J. 1998. A critical role of natural immunoglobulin $M$ in immediate defense against systemic bacterial infection. J. Exp. Med. 188:2381-2386.

67. Baumgarth, N., et al. 1999. Innate and acquired humoral immunities to influenza virus are mediated by distinct arms of the immune system. Proc. Natl. Acad. Sci. U. S. A. 96:2250-2255.

68. Lutz, H.U. 2007. Homeostatic roles of naturally occurring antibodies: an overview. J. Autoimmun. 29:287-294.

69. Boes, M., et al. 2000. Accelerated development of IgG autoantibodies and autoimmune disease in the absence of secreted IgM. Proc. Natl. Acad. Sci. U. S. A. 97:1184-1189.

70. Quartier, P., Potter, P.K., Ehrenstein, M.R., Walport, M.J., and Botto, M. 2005. Predominant role of IgMdependent activation of the classical pathway in the clearance of dying cells by murine bone marrow-derived macrophages in vitro. Eur. J. Immunol. 35:252-260.

71. Ogden, C.A., Kowalewski, R., Peng, Y., Montenegro, V., and Elkon, K.B. 2005. IGM is required for efficient complement mediated phagocytosis of apoptotic cells in vivo. Autoimmunity. 38:259-264.

72. Kearney,J.F., Barletta, R., Quan, Z.S., and Quintans, J. 1981. Monoclonal vs. heterogeneous anti-H-8 antibodies in the analysis of the anti-phosphorylcholine response in BALB/c mice. Eur. J. Immunol. 11:877-883.

73. Xu, D., et al. 1997. Epitope characterization of malondialdehyde-acetaldehyde adducts using an enzyme-linked immunosorbent assay. Chem. Res. Toxicol. 10:978-986. 